\title{
The Virasoro fusion kernel and Ruijsenaars' hypergeometric function
}

\author{
Julien Roussillon ${ }^{1}$
}

Received: 2 July 2020 / Revised: 1 November 2020 / Accepted: 22 December 2020 /

Published online: 9 January 2021

(c) The Author(s) 2021

\begin{abstract}
We show that the Virasoro fusion kernel is equal to Ruijsenaars' hypergeometric function up to normalization. More precisely, we prove that the Virasoro fusion kernel is a joint eigenfunction of four difference operators. We find a renormalized version of this kernel for which the four difference operators are mapped to four versions of the quantum relativistic hyperbolic Calogero-Moser Hamiltonian tied with the root system $B C_{1}$. We consequently prove that the renormalized Virasoro fusion kernel and the corresponding quantum eigenfunction, the (renormalized) Ruijsenaars hypergeometric function, are equal.
\end{abstract}

Keywords $2 \mathrm{~d}$ conformal field theory · Integrability

Mathematics Subject Classification $81 \mathrm{~T} 40 \cdot 81 \mathrm{Q} 80$

\section{Contents}

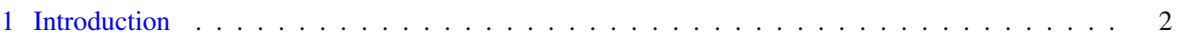

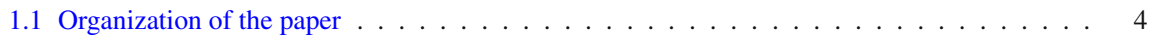

2 Ruijsenaars' hypergeometric function . . . . . . . . . . . . . . . . . 4

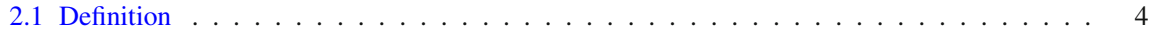

2.2 Symmetry properties . . . . . . . . . . . . . . . . . . . . 5

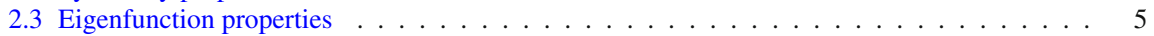

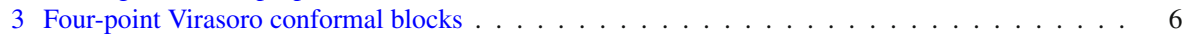

3.1 Highest-weight representations of the Virasoro algebra . . . . . . . . . . . . . . . 6

3.2 Four-point Virasoro conformal blocks . . . . . . . . . . . . . . . . . . . 7

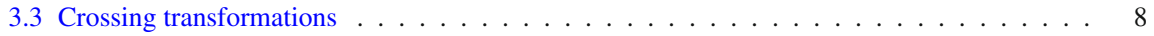

4 The Virasoro fusion kernel . . . . . . . . . . . . . . . . . . . . . . . . . . 9

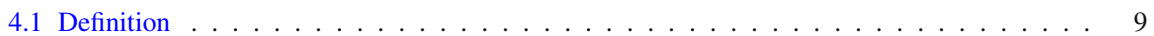

4.2 Symmetry properties . . . . . . . . . . . . . . . . . . . . 10

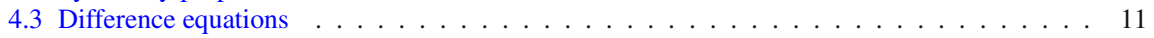

Julien Roussillon

julienro@kth.se

1 Department of Mathematics, KTH Royal Institute of Technology, 10044 Stockholm, Sweden 
4.3.1 First pair of difference equations . . . . . . . . . . . . . . . . . . . . 12

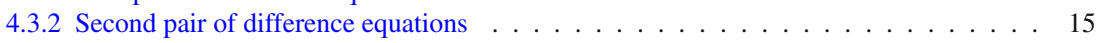

5 Main result . . . . . . . . . . . . . . . . . . . . . . . . . 17

5.1 Ruijsenaars/CFT parameter correspondence . . . . . . . . . . . . . . . . . . . 17

5.2 Renormalized fusion kernel $F_{\text {ren }} \ldots \ldots \ldots \ldots \ldots$. . . . . . . . . . . 18

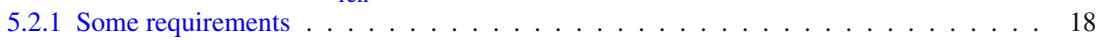

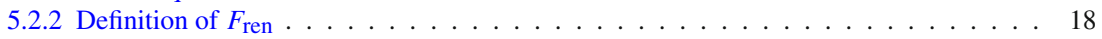

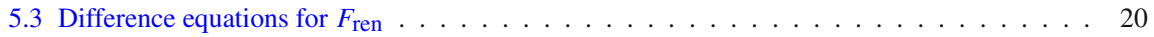

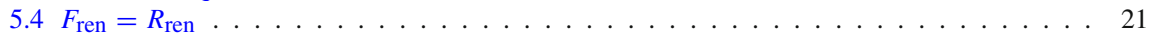

6 Conclusion and perspectives . . . . . . . . . . . . . . . . . . . . 23

Appendix A. Special functions . . . . . . . . . . . . . . . . . . . . . . . . . . . 24

Appendix B. Hyperbolic Barnes integral . . . . . . . . . . . . . . . . . . . . . . . 26

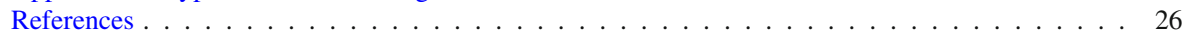

\section{Introduction}

Two-dimensional conformal field theories (CFTs) have been intensively studied since the seminal work of Belavin et al. [3]. In addition to their broad range of physical applications in, for example, condensed matter theory and string theory, they possess rich underlying mathematical structures.

The infinite-dimensional symmetry algebra of 2D CFTs determines the structure of their correlation functions. The operator product expansion makes it possible to decompose $\mathrm{N}$-point correlators on the Riemann sphere into combinations of threepoint structure constants and universal quantities called Virasoro conformal blocks. In particular, there are three ways to decompose the four-point correlation function. The universal parts of each decomposition are called $s-, t$ - and $u$-channel conformal blocks. Since each decomposition must lead to the same four-point function, the three kinds of conformal blocks are related by duality transformations.

In particular, the $s$ - and $t$-channel conformal blocks are related by an integral transform called fusion transformation. Such a transformation was conjectured in [21] and established in [36]. The corresponding kernel, the Virasoro fusion kernel, was constructed by Ponsot and Teschner [21,22] and revisited in [38] as b-6j symbols for the modular double of $\mathcal{U}_{q}\left(s l_{2}(\mathbb{R})\right)$. This terminology comes from the fact that $b$, which is related to the central charge of the Virasoro algebra by $c=1+6\left(b+b^{-1}\right)^{2}$, is associated with two unimodular parameters $q=e^{i \pi b^{2}}$ and $\tilde{q}=e^{i \pi b^{-2}}$. The Virasoro fusion kernel also appears in quantum Teichmüller theory [20] and in 3d supersymmetric gauge theories [38,39], and recent physical applications were found in $[6,7,12]$.

The Virasoro fusion kernel is denoted $F\left(b, \boldsymbol{\theta}, \sigma_{s}, \sigma_{t}\right)$. Here, $\boldsymbol{\theta}$ is a set of four external conformal dimensions associated with the fields entering the four-point correlation function, while $\sigma_{s}$ and $\sigma_{t}$ are two internal conformal dimensions associated with the $s$ - and $t$-channel decompositions of the four-point function.

The Ponsot-Teschner formula [21] for $F$ is recalled in (4.1). It is given by a contour integral whose integrand involves a special function $s_{b}(z)$. The $s_{b}$-function appears in several different contexts. It was introduced under the names of "Quantum Dilogarithm function," "Hyperbolic Gamma function" and "Quantum Exponential function" in $[9,25,41]$, respectively. ${ }^{1}$ In particular, $F$ is proportional to a hyperbolic Barnes inte-

\footnotetext{
${ }^{1}$ The hyperbolic gamma function first appeared in [24] but was named as such in [25].
} 
gral which is a hyperbolic generalization of the Barnes representation for the Gauss hypergeometric function. Such an integral was constructed in [5] as a degeneration limit of the hyperbolic hypergeometric function, and it also arises as a limit of Spiridonov's elliptic hypergeometric $V$-function $[32,38]$. Finally, the Virasoro fusion kernel is a joint eigenfunction of four difference operators [17,21].

On the other hand, Calogero-Moser (CM) models are systems of particles living on the real line or the circle and interacting with a rational, trigonometric/hyperbolic or elliptic potential. Their relativistic deformations were found by Ruijsenaars and Schneider at the classical level [31]. Quantum relativistic CM systems tied with the root system $A_{N-1}$ were found by Ruijsenaars [30], and their $B C_{N}$ generalization by van Diejen [40]. Therefore, we will refer to quantum relativistic $B C_{N} \mathrm{CM}$ systems as quantum Ruijsenaars-van Diejen (RvD) systems. In this article, only the case $N=1$ of the latter system will be considered.

The rank $N$ quantum trigonometric RvD system is solved by the Koornwinder $N$-variable polynomials [13]. However, in the hyperbolic case the eigenfunctions are non-polynomial and are only known in the rank one. The corresponding eigenfunction, the Ruijsenaars hypergeometric function, was introduced in [24] and studied in greater detail in [26-28]. This function is denoted $R\left(a_{-}, a_{+}, \gamma, v, \hat{v}\right)$; here, $a_{-}$and $a_{+}$are associated with two unimodular quantum deformation parameters $q=e^{i \pi a_{-} / a_{+}}$and $\tilde{q}=e^{i \pi a_{+} / a_{-}}$, while $\boldsymbol{\gamma}$ is a set of four external couplings constants. Finally, $v$ and $\hat{v}$ are viewed as geometric and spectral variables, respectively.

Of particular importance is the renormalized $R$-function, denoted $R_{\text {ren }}\left(a_{-}, a_{+}, \gamma, v, \hat{v}\right)$, which was defined in [26]. The definition (2.3) and the properties of the function $R_{\text {ren }}$ resemble those of the Virasoro fusion kernel. First of all, $R_{\text {ren }}$ is defined as a contour integral whose integrand involves the hyperbolic gamma function $G\left(a_{-}, a_{+}, z\right)$. The functions $G$ and $s_{b}$ are simply related by

$$
s_{b}(z)=G\left(b, b^{-1}, z\right)
$$

$R_{\text {ren }}$ is also proportional to a hyperbolic Barnes integral [5]. Moreover, it is a joint eigenfunction of four difference operators which are four versions of the rank one quantum hyperbolic RvD Hamiltonian. Let us finally mention that the function $R_{\text {ren }}$ was related to the modular double of $\mathcal{U}_{q}\left(s l_{2}(\mathbb{R})\right)$ in [4].

The discussion above suggests that the Virasoro fusion kernel and the Ruijsenaars hypergeometric function are closely related. The aim of this article is to show that the two functions are equal up to normalization (see Theorem 1). The identification procedure can be summarized as follows. We prove in Propositions 4.3 and 4.4 that the Virasoro fusion kernel is a joint eigenfunction of four difference operators. We provide the Ruijsenaars/CFT parameters identification in Sect. 5.1. We define in (5.14) a renormalized version $F_{\text {ren }}$ of $F$, and we show in Proposition 5.3 that both $R_{\text {ren }}$ and $F_{\text {ren }}$ satisfy the same four difference equations. Therefore, the two functions are proportional. We finally show in Theorem 1 that they are actually equal: The proof is rather simple and follows from the identity (B.4) satisfied by the hyperbolic Barnes integral. 


\subsection{Organization of the paper}

The Ruijsenaars hypergeometric function and various of its properties are recalled in Sect. 2. Section 3 reviews some properties of the four-point Virasoro conformal blocks and introduces the Virasoro fusion kernel in this context. In Sect. 4, we study various symmetry and eigenfunction properties of $F$. Finally, our main result-the identification of $F$ with $R_{\text {ren }}$-is presented in Sect. 5.

\section{Ruijsenaars' hypergeometric function}

This section is a brief overview of the (renormalized) Ruijsenaars hypergeometric function following [26]. We recall several of its properties which we will need to relate it to the Virasoro fusion kernel.

\subsection{Definition}

Define two sets of external couplings constants $\gamma$ and $\hat{\gamma}$ by

$$
\boldsymbol{\gamma}=\left(\begin{array}{l}
\gamma_{0} \\
\gamma_{1} \\
\gamma_{2} \\
\gamma_{3}
\end{array}\right), \quad \hat{\gamma}=\left(\begin{array}{l}
\hat{\gamma}_{0} \\
\hat{\gamma}_{1} \\
\hat{\gamma}_{2} \\
\hat{\gamma}_{3}
\end{array}\right)=J \boldsymbol{\gamma},
$$

where $J$ is a matrix satisfying $J^{2}=\mathcal{I}_{4}$ and is defined by

$$
J=\frac{1}{2}\left(\begin{array}{cccc}
1 & 1 & 1 & 1 \\
1 & 1 & -1 & -1 \\
1 & -1 & 1 & -1 \\
1 & -1 & -1 & 1
\end{array}\right) .
$$

The renormalized $R$-function denoted $R_{\text {ren }}\left(a_{-}, a_{+}, \gamma, v, \hat{v}\right)$ is given by a contour integral whose integrand involves the hyperbolic gamma function $G\left(a_{-}, a_{+}, z\right)$. The definition of $G$ and its properties can be found in Appendix A. Its dependence on $a_{-}$ and $a_{+}$will be omitted for simplicity. Following [26], the function $R_{\text {ren }}$ is defined for $\left(a_{-}, a_{+}, \boldsymbol{\gamma}\right) \in \mathrm{RHP}^{2} \times \mathbb{C}^{4}$, where RHP denotes the open right-half plane, such that

$$
\begin{gathered}
R_{\mathrm{ren}}\left(a_{-}, a_{+}, \boldsymbol{\gamma}, v, \hat{v}\right)=\frac{1}{\sqrt{a_{+} a_{-}}} \prod_{\epsilon= \pm} \frac{G\left(\epsilon v-i \gamma_{0}\right)}{G\left(\epsilon \hat{v}+i \hat{\gamma}_{0}\right)} \\
\int_{\mathrm{R}} d z \frac{\prod_{\epsilon= \pm} G\left(z+\epsilon v+i \gamma_{0}\right) G\left(z+\epsilon \hat{v}+i \hat{\gamma}_{0}\right)}{G(z+i a) \prod_{j=1}^{3} G\left(z+i \gamma_{0}+i \gamma_{j}+i a\right)},
\end{gathered}
$$

and where $a=\left(a_{+}+a_{-}\right) / 2$. The function $G$ has sequences of poles and zeros given in (A.4) and (A.5), respectively. Therefore, for instance, when $a_{+}, a_{-}>0$ the integrand in (2.3) has eight semi-infinite lines of poles, four of them increasing and the other ones 
decreasing. The integration contour $\mathrm{R}$ runs from $-\infty$ to $+\infty$ separating the increasing and decreasing sequences of poles. Moreover, for fixed values of $a_{-}, a_{+}$and $\gamma$, the function $R_{\text {ren }}$ is meromorphic in $v$ and $\hat{v}$.

Finally, it was shown in [5, Proposition 4.20] that the function $R_{\text {ren }}$ is proportional to a hyperbolic Barnes integral $\mathcal{B}_{h}\left(a_{-}, a_{+}, \boldsymbol{u}\right)$ whose definition is recalled in Appendix B. $\mathcal{B}_{h}\left(a_{-}, a_{+}, \boldsymbol{u}\right)$ is one possible degeneration of the hyperbolic hypergeometric function, and other types of degenerations led the authors of [5] to find other representations for $R_{\text {ren. }}$.

\subsection{Symmetry properties}

The function $R_{\text {ren }}$ possesses various discrete symmetries which follow from the representation (2.3). In view of the property (A.6) of the hyperbolic gamma function, $R_{\text {ren }}$ is scale invariant:

$$
R_{\mathrm{ren}}\left(\lambda a_{-}, \lambda a_{+}, \lambda \gamma, \lambda v, \lambda \hat{v}\right)=R_{\mathrm{ren}}\left(a_{-}, a_{+}, \boldsymbol{\gamma}, v, \hat{v}\right), \quad \lambda>0
$$

Moreover, because of the fact that $G\left(a_{-}, a_{+}, z\right)=G\left(a_{+}, a_{-}, z\right)$, the following identity holds:

$$
R_{\text {ren }}\left(a_{-}, a_{+}, \gamma, v, \hat{v}\right)=R_{\text {ren }}\left(a_{+}, a_{-}, \gamma, v, \hat{v}\right) \quad \text { (modular symmetry) } .
$$

It can also be verified that $R_{\text {ren }}$ satisfies

$$
R_{\mathrm{ren}}\left(a_{-}, a_{+}, \boldsymbol{\gamma}, v, \hat{v}\right)=R_{\mathrm{ren}}\left(a_{-}, a_{+}, \hat{\boldsymbol{\gamma}}, \hat{v}, v\right) \quad \text { (self-duality) }
$$

Finally, the function $R_{\text {ren }}$ is even in $v, \hat{v}$ and is symmetric under permutations of $\gamma_{1}, \gamma_{2}, \gamma_{3}$. It was shown in [27, Theorem 1.1] that a similarity transformed version of $R_{\text {ren }}$ has an extended $D_{4}$-symmetry in $\boldsymbol{\gamma}$. More precisely, define the function $\mathcal{E}_{\text {ren }}$ by

$$
\mathcal{E}_{\text {ren }}\left(a_{-}, a_{+}, \boldsymbol{\gamma}, v, \hat{v}\right)=\frac{R_{\mathrm{ren}}\left(a_{-}, a_{+}, \boldsymbol{\gamma}, v, \hat{v}\right)}{\delta\left(a_{-}, a_{+}, \boldsymbol{\gamma}, v\right) \delta\left(a_{-}, a_{+}, \hat{\boldsymbol{\gamma}}, \hat{v}\right)},
$$

where the function $\delta$ is given by

$$
\delta\left(a_{-}, a_{+}, \boldsymbol{\gamma}, v\right)=\frac{\prod_{\mu=0}^{3} G\left(v-i \gamma_{\mu}\right)}{G(2 v+i a)} .
$$

The function $\mathcal{E}_{\text {ren }}$ is invariant under all permutations of $\gamma_{0}, \gamma_{1}, \gamma_{2}, \gamma_{3}$ and under flipping the sign of any pair of $\gamma_{\mu}$ 's. The resulting invariance group is the Weyl group of the Lie algebra $D_{4}$.

\subsection{Eigenfunction properties}

Define a translation operator $e^{ \pm i a_{-} \partial_{x}}$ which formally acts on meromorphic functions $f(x)$ as $e^{ \pm i a_{-} \partial_{x}} f(x)=f\left(x \pm i a_{-}\right)$. The quantum relativistic Calogero-Moser Hamil- 
tonian tied with the root system $B C_{1}$ and with a hyperbolic interaction was defined in [40]. As mentioned in the introduction, it will be referred to as the Ruijsenaars-van Diejen (RvD) Hamiltonian. It is a difference operator of the form

$$
H_{R v D}\left(a_{-}, a_{+}, \boldsymbol{\gamma}, x\right)=C\left(a_{-}, a_{+}, \boldsymbol{\gamma}, x\right) e^{-i a_{-} \partial_{x}}+C\left(a_{-}, a_{+}, \boldsymbol{\gamma},-x\right) e^{i a_{-} \partial_{x}}+V\left(a_{-}, a_{+}, \boldsymbol{\gamma}, x\right),
$$

where

$$
C\left(a_{-}, a_{+}, \boldsymbol{\gamma}, x\right)=-\frac{4 \prod_{k=0}^{3} \cosh \left(\frac{\pi}{a_{+}}\left(x-i \gamma_{k}-\frac{i a_{-}}{2}\right)\right)}{\sinh \left(\frac{2 \pi x}{a_{+}}\right) \sinh \left(\frac{2 \pi}{a_{+}}\left(x-\frac{i a_{-}}{2}\right)\right)},
$$

and where the potential $V$ is defined by

$$
V\left(a_{-}, a_{+}, \boldsymbol{\gamma}, x\right)=-C\left(a_{-}, a_{+}, \boldsymbol{\gamma}, x\right)-C\left(a_{-}, a_{+}, \boldsymbol{\gamma},-x\right)-2 \cos \left(\frac{\pi}{a_{+}}\left(\sum_{\mu=0}^{3} \gamma_{\mu}+a_{-}\right)\right) .
$$

The function $R_{\text {ren }}$ is a joint eigenfunction of four versions of this Hamiltonian [26, Theorem 3.1]. More precisely, for $\left(a_{-}, a_{+}, \boldsymbol{\gamma}, v, \hat{v}\right) \in \mathrm{RHP}^{2} \times \mathbb{C}^{6}$ the following eigenvalue equations hold:

$$
\begin{aligned}
& H_{R v D}\left(a_{-}, a_{+}, \boldsymbol{\gamma}, v\right) R_{\mathrm{ren}}\left(a_{-}, a_{+}, \boldsymbol{\gamma}, v, \hat{v}\right)=2 \cosh \left(\frac{2 \pi \hat{v}}{a_{+}}\right) R_{\mathrm{ren}}\left(a_{-}, a_{+}, \boldsymbol{\gamma}, v, \hat{v}\right), \\
& H_{R v D}\left(a_{+}, a_{-}, \boldsymbol{\gamma}, v\right) R_{\mathrm{ren}}\left(a_{-}, a_{+}, \boldsymbol{\gamma}, v, \hat{v}\right)=2 \cosh \left(\frac{2 \pi \hat{v}}{a_{-}}\right) R_{\mathrm{ren}}\left(a_{-}, a_{+}, \boldsymbol{\gamma}, v, \hat{v}\right), \\
& H_{R v D}\left(a_{-}, a_{+}, \hat{\boldsymbol{\gamma}}, \hat{v}\right) R_{\mathrm{ren}}\left(a_{-}, a_{+}, \boldsymbol{\gamma}, v, \hat{v}\right)=2 \cosh \left(\frac{2 \pi v}{a_{+}}\right) R_{\mathrm{ren}}\left(a_{-}, a_{+}, \boldsymbol{\gamma}, v, \hat{v}\right), \\
& H_{R v D}\left(a_{+}, a_{-}, \hat{\boldsymbol{\gamma}}, \hat{v}\right) R_{\mathrm{ren}}\left(a_{-}, a_{+}, \boldsymbol{\gamma}, v, \hat{v}\right)=2 \cosh \left(\frac{2 \pi v}{a_{-}}\right) R_{\mathrm{ren}}\left(a_{-}, a_{+}, \boldsymbol{\gamma}, v, \hat{v}\right) .
\end{aligned}
$$

It can be noted that $(2.12 \mathrm{a})$ implies $(2.12 \mathrm{~b}),(2.12 \mathrm{c})$ and $(2.12 \mathrm{~d})$ thanks to the properties (2.5) and (2.6) of $R_{\text {ren }}$.

\section{Four-point Virasoro conformal blocks}

In this section, we briefly review the four-point Virasoro conformal blocks and recall how the Virasoro fusion kernel arises in this context. A more complete overview of the subject can be found in $[23,35,36]$.

\subsection{Highest-weight representations of the Virasoro algebra}

The Virasoro algebra is the symmetry algebra of two-dimensional conformal field theories. It has generators $L_{n}, n \in \mathbb{Z}$ and relations

$$
\left[L_{n}, L_{m}\right]=(n-m) L_{n+m}+\frac{c}{12} n\left(n^{2}-1\right) \delta_{n+m, 0}
$$


where $c$ is a central element in the algebra called central charge. Highest-weight representations $\mathcal{V}_{\theta}$ of the Virasoro algebra are generated from vectors $\mid \theta>$ which satisfy

$$
L_{0}|\theta>=\Delta(\theta)| \theta>, \quad L_{n} \mid \theta>=0, \quad n>0,
$$

where a Liouville-type parameterization of $\Delta$ and $c$ is used:

$$
\Delta(x)=\frac{Q^{2}}{4}+x^{2}, \quad c=1+6 Q^{2}, \quad Q=b+b^{-1} .
$$

The representations $\mathcal{V}_{\theta}$ decompose as follows:

$$
\mathcal{V}_{\theta} \simeq \bigoplus_{n \in \mathbb{Z} \geq 0} \mathcal{V}_{\theta}^{(n)}
$$

where $L_{0} v=(\Delta(\theta)+n) v$ for all $v \in \mathcal{V}_{\theta}^{(n)}$. A chiral vertex operator $V_{\Delta\left(\theta_{2}\right), \Delta\left(\theta_{1}\right)}^{\Delta\left(\theta_{0}\right)}(z)$ is a map $V_{\Delta\left(\theta_{2}\right), \Delta\left(\theta_{1}\right)}^{\Delta\left(\theta_{0}\right)}: \mathcal{V}_{\theta_{1}} \rightarrow \mathcal{V}_{\theta_{2}}$. It is defined by the following commutation relations:

$$
\left[L_{n}, V_{\Delta\left(\theta_{2}\right), \Delta\left(\theta_{1}\right)}^{\Delta\left(\theta_{0}\right)}(z)\right]=z^{n}\left(z \partial_{z}+\Delta\left(\theta_{0}\right)(n+1)\right) V_{\Delta\left(\theta_{2}\right), \Delta\left(\theta_{1}\right)}^{\Delta\left(\theta_{0}\right)}(z)
$$

In particular, it admits the following formal series expansion:

$$
V_{\Delta\left(\theta_{2}\right), \Delta\left(\theta_{1}\right)}^{\Delta\left(\theta_{0}\right)}(z) \mid \theta_{1}>=z^{\Delta\left(\theta_{2}\right)-\Delta\left(\theta_{1}\right)-\Delta\left(\theta_{0}\right)} \sum_{n \in \mathbb{Z}_{\geq 0}} v_{n} z^{n},
$$

where $v_{n} \in \mathcal{V}_{\theta_{2}}$ and $v_{0}=\mid \theta_{2}>$. We emphasize that the expansion (3.6) can be renormalized by an arbitrary factor $N\left(\theta_{2}, \theta_{0}, \theta_{1}\right)$. Such a factor will be introduced in Sect. 5.

\subsection{Four-point Virasoro conformal blocks}

We now define a set of external conformal dimensions $\theta$ by

$$
\boldsymbol{\theta}=\left(\begin{array}{c}
\theta_{0} \\
\theta_{t} \\
\theta_{1} \\
\theta_{\infty}
\end{array}\right)
$$

The four-point Virasoro conformal block is defined by the expectation value of a composition of two chiral vertex operators as follows:

$$
\mathcal{F}\left(b, \boldsymbol{\theta}, \sigma_{s} ; z\right)=\left\langle\theta_{\infty}\left|V_{\Delta\left(\theta_{\infty}\right), \Delta\left(\sigma_{s}\right)}^{\Delta\left(\theta_{1}\right)}(1) V_{\Delta\left(\sigma_{s}\right), \Delta\left(\theta_{0}\right)}^{\Delta\left(\theta_{t}\right)}(z)\right| \theta_{0}\right\rangle
$$


The parameters $\boldsymbol{\theta}$ and $\sigma_{s}$ will be referred to as external and internal momenta. The block $\mathcal{F}$ admits a series expansion in $z$ which can be computed recursively using (3.1), (3.5) and (3.6):

$$
\mathcal{F}\left(b, \boldsymbol{\theta}, \sigma_{s} ; z\right)=z^{\Delta\left(\sigma_{s}\right)-\Delta\left(\theta_{0}\right)-\Delta\left(\theta_{t}\right)}\left(1+\frac{\left(\Delta\left(\sigma_{s}\right)+\Delta\left(\theta_{1}\right)-\Delta\left(\theta_{\infty}\right)\right)\left(\Delta\left(\sigma_{s}\right)+\Delta\left(\theta_{t}\right)-\Delta\left(\theta_{0}\right)\right)}{2 \Delta\left(\sigma_{s}\right)} z+\sum_{k=2}^{\infty} c_{k} z^{k}\right)
$$

The discovery of the AGT relation [2] between two-dimensional conformal field theories and four-dimensional supersymmetric gauge theories led to a closed-form expression for all the coefficients $c_{k}$. In our notation, the complete expansion of (3.9) can be found in [15, Eq. (3.1)]. Several conjectures exist for the analytic properties of four-point Virasoro conformal blocks. The series in (3.9) is believed to be convergent inside the unit disk $|z|<1$. Moreover, the only singularities of the conformal blocks as a function of $z$ are expected to be branch points at $0,1, \infty[10,42]$. Under this assumption, conformal blocks are naturally defined for $z \in \mathbb{C} \backslash((-\infty, 0] \cup[1, \infty))$. Finally, they are believed to be analytic in $\boldsymbol{\theta}$ and meromorphic in $\sigma_{s}$, with the only possible poles located at $\pm \sigma_{s}^{(m, n)}=-\frac{i}{2}\left(m b+n b^{-1}\right)$ for $m, n \in \mathbb{Z}_{>0}$.

Let us finally mention that $\mathcal{F}$ admits another representation called Zamolodchikov's recursion [42]. This representation is a power series of the nome

$$
q(z)=\exp \left(-\pi \frac{{ }_{2} F_{1}\left(\frac{1}{2}, \frac{1}{2}, 1,1-z\right)}{{ }_{2} F_{1}\left(\frac{1}{2}, \frac{1}{2}, 1, z\right)}\right)
$$

where ${ }_{2} F_{1}(a, b, c, z)$ is the hypergeometric function. Whereas the representation (3.9) is expected to converge for $|z|<1$, Zamolodchikov's recursion converges faster than (3.9) and for all $z \in \mathbb{C} \backslash\{1\}$.

\subsection{Crossing transformations}

The linear span (3.9) of four-point Virasoro conformal blocks with different internal dimensions $\sigma_{s}$ forms an infinite-dimensional representation of $\Gamma\left(\Sigma_{0,4}\right)=\operatorname{PSL}_{2}(\mathbb{Z})$, the mapping class group of the four-puncture Riemann sphere. It is generated by the braiding $B$ and fusion $F$ moves, such that $F^{2}=(B F)^{3}=1$. The three ways of splitting four points on $\mathbb{C P}^{1}$ into two pairs define the $s-, t$ - and $u$-channel bases for the space of conformal blocks. The cross-ratio argument of conformal blocks in these channels is chosen from $\left\{z, \frac{z}{z-1}\right\},\left\{1-z, \frac{z-1}{z}\right\}$, and $\left\{\frac{1}{z}, \frac{1}{1-z}\right\}$, respectively. The braiding move $B$ acts on the $s$-channel conformal blocks as follows:

$\mathcal{F}\left(b, \boldsymbol{\theta}, \sigma_{s} ; z\right)=e^{ \pm i \pi\left(\Delta\left(\sigma_{s}\right)-\Delta\left(\theta_{0}\right)-\Delta\left(\theta_{t}\right)\right)}(1-z)^{-2 \Delta\left(\theta_{t}\right)} \mathcal{F}\left(b, \tilde{\boldsymbol{\theta}}, \sigma_{s} ; \frac{z}{z-1}\right), \quad \operatorname{Im} z \gtrless 0$,

where $\tilde{\boldsymbol{\theta}}$ is obtained by performing the permutation $\theta_{1} \leftrightarrow \theta_{\infty}$ on $\boldsymbol{\theta}$. On the other hand, the fusion move was conjectured in [21] and established in [36]. It is represented by 
the integral transform

$$
\mathcal{F}\left(b, \boldsymbol{\theta}, \sigma_{s} ; z\right)=\int_{\mathbb{R}_{+}} d \sigma_{t} F\left(b, \boldsymbol{\theta}, \sigma_{s}, \sigma_{t}\right) \mathcal{F}\left(b, \hat{\boldsymbol{\theta}}, \sigma_{t} ; 1-z\right),
$$

where $\hat{\boldsymbol{\theta}}$ is obtained by performing the permutation $\theta_{0} \leftrightarrow \theta_{1}$ on $\boldsymbol{\theta}$. It will be convenient to explicitly write $\hat{\boldsymbol{\theta}}$ as

$$
\hat{\boldsymbol{\theta}}=K \boldsymbol{\theta}, \quad K=\left(\begin{array}{llll}
0 & 0 & 1 & 0 \\
0 & 1 & 0 & 0 \\
1 & 0 & 0 & 0 \\
0 & 0 & 0 & 1
\end{array}\right)
$$

Finally, the kernel $F\left(b, \boldsymbol{\theta}, \sigma_{s}, \sigma_{t}\right)$ of the fusion move is the Virasoro fusion kernel.

\section{The Virasoro fusion kernel}

In this section, we recall the Ponsot-Teschner formula [21] for the Virasoro fusion kernel. Let us also note that other representations for $F$ were found in [38]. Moreover, we describe various symmetry and eigenfunction properties of $F$ which will be useful in its identification with the Ruijsenaars hypergeometric function (2.3).

\subsection{Definition}

The Virasoro fusion kernel is defined by a contour integral involving two special functions $s_{b}(x)=g_{b}(x) / g_{b}(-x)$ and $g_{b}(x)$ which are closely related to the hyperbolic gamma function $G\left(a_{-}, a_{+}, x\right)=E\left(a_{-}, a_{+}, x\right) / E\left(a_{-}, a_{+},-x\right)$ and $E\left(a_{-}, a_{+}, x\right)$, respectively. The exact relations are given in (A.9). The Ponsot-Teschner formula reads

$$
\begin{aligned}
F\left(b, \boldsymbol{\theta}, \sigma_{s}, \sigma_{t}\right)= & \prod_{\epsilon, \epsilon^{\prime}= \pm} \frac{g_{b}\left(\epsilon \theta_{1}+\theta_{t}+\epsilon^{\prime} \sigma_{t}\right) g_{b}\left(\epsilon \theta_{0}-\theta_{\infty}+\epsilon^{\prime} \sigma_{t}\right)}{g_{b}\left(\epsilon \theta_{0}+\theta_{t}+\epsilon^{\prime} \sigma_{s}\right) g_{b}\left(\epsilon \theta_{1}-\theta_{\infty}+\epsilon^{\prime} \sigma_{s}\right)} \prod_{\epsilon= \pm} \frac{g_{b}\left(\frac{i Q}{2}+2 \epsilon \sigma_{s}\right)}{g_{b}\left(-\frac{i Q}{2}+2 \epsilon \sigma_{t}\right)} \\
& \times \int_{\mathrm{F}} \mathrm{d} x \prod_{\epsilon= \pm 1} \frac{s_{b}\left(x+\epsilon \theta_{1}\right) s_{b}\left(x+\epsilon \theta_{0}+\theta_{\infty}+\theta_{t}\right)}{s_{b}\left(x+\frac{i Q}{2}+\theta_{\infty}+\epsilon \sigma_{s}\right) s_{b}\left(x+\frac{i Q}{2}+\theta_{t}+\epsilon \sigma_{t}\right)} .
\end{aligned}
$$

When $b>0$, the integrand in (4.1) has eight vertical semi-infinite lines of poles, four of them increasing and the other four decreasing; the integration contour $F$ runs from $-\infty$ to $+\infty$, separating the increasing and decreasing sequences of poles. More generally, the fusion kernel (4.1) can be extended to a meromorphic function of all of its parameters provided that $c \in \mathbb{C} \backslash \mathbb{R}_{\leq 1}$, which corresponds to $b \notin i \mathbb{R}$. 


\subsection{Symmetry properties}

We now present various symmetry properties of the Virasoro fusion kernel. First, because the Virasoro conformal block (3.9) is a function of the conformal dimensions $\Delta(x)=\frac{Q^{2}}{4}+x^{2}$, it follows that it is even in $\boldsymbol{\theta}$ and $\sigma_{s}$. Therefore, the fusion transformation (3.11) implies that the Virasoro fusion kernel $F\left(b, \boldsymbol{\theta}, \sigma_{s}, \sigma_{t}\right)$ is even in $\boldsymbol{\theta}$ and $\sigma_{s}, \sigma_{t}$. Second, because $s_{b}(x)=s_{b^{-1}}(x)$ and $g_{b}(x)=g_{b^{-1}}(x), F$ has the following symmetry:

$$
F\left(b, \boldsymbol{\theta}, \sigma_{s}, \sigma_{t}\right)=F\left(b^{-1}, \boldsymbol{\theta}, \sigma_{s}, \sigma_{t}\right) .
$$

We now describe a duality transformation exchanging the internal momenta $\sigma_{s}$ and $\sigma_{t}$.

Proposition 4.1 The Virasoro fusion kernel given in (4.1) satisfies

$$
F\left(b, \boldsymbol{\theta}, \sigma_{s}, \sigma_{t}\right)=\frac{\alpha\left(\boldsymbol{\theta}, \sigma_{s}\right) \alpha\left(\boldsymbol{\theta},-\sigma_{s}\right)}{\alpha\left(\hat{\boldsymbol{\theta}}, \sigma_{t}\right) \alpha\left(\hat{\boldsymbol{\theta}},-\sigma_{t}\right)} F\left(b, \hat{\boldsymbol{\theta}}, \sigma_{t}, \sigma_{s}\right),
$$

where $\hat{\boldsymbol{\theta}}$ is related to $\boldsymbol{\theta}$ as in (3.12) and

$$
\alpha\left(\boldsymbol{\theta}, \sigma_{s}\right)=\frac{g_{b}\left(2 \sigma_{s}+\frac{i Q}{2}\right) g_{b}\left(2 \sigma_{s}-\frac{i Q}{2}\right)}{\prod_{\epsilon, \epsilon^{\prime}= \pm} g_{b}\left(\sigma_{s}+\epsilon \theta_{1}+\epsilon^{\prime} \theta_{\infty}\right) g_{b}\left(\sigma_{s}+\epsilon \theta_{0}+\epsilon^{\prime} \theta_{t}\right)} .
$$

Proof The proof relies on the fact that the contour integral in (4.1) is a hyperbolic Barnes integral $\mathcal{B}_{h}$ (see (B.2) for its definition). In particular, $\mathcal{B}_{h}$ satisfies the remarkable identity (B.4) which is crucial for the proof.

Following the definition (B.2) and the relation $s_{b}(z)=G\left(b, b^{-1}, z\right)$, it is easy to verify that the contour integral in (4.1) takes the form

$$
\int_{\mathrm{F}} \mathrm{d} x \prod_{\epsilon= \pm} \frac{s_{b}\left(x+\epsilon \theta_{1}\right) s_{b}\left(x+\epsilon \theta_{0}+\theta_{\infty}+\theta_{t}\right)}{s_{b}\left(x+\frac{i Q}{2}+\theta_{\infty}+\epsilon \sigma_{s}\right) s_{b}\left(x+\frac{i Q}{2}+\theta_{t}+\epsilon \sigma_{t}\right)}=\frac{1}{2} \mathcal{B}_{h}\left(b, b^{-1}, \boldsymbol{u}\right),
$$

where $\boldsymbol{u} \in \mathcal{G}_{i} Q$ and $\mathcal{G}_{k}$ is defined by (B.1). Various choices for the eight parameters $\boldsymbol{u}$ lead to the same contour integral. Here, we choose

$$
\begin{aligned}
& u_{1}=\frac{i Q}{2}+\theta_{\infty}+\sigma_{s}, \quad u_{2}=\frac{i Q}{2}+\theta_{\infty}-\sigma_{s}, \quad u_{3}=\theta_{1}, \quad u_{4}=-\theta_{1}, \\
& u_{5}=-\theta_{0}-\theta_{\infty}-\theta_{t}, \quad u_{6}=\theta_{0}-\theta_{\infty}-\theta_{t}, \quad u_{7}=\frac{i Q}{2}+\theta_{t}-\sigma_{t}, \quad u_{8}=\frac{i Q}{2}+\theta_{t}+\sigma_{t} .
\end{aligned}
$$

We are now going to apply the identity (B.4) satisfied by the hyperbolic Barnes integral. First, from (B.3) the action of $\omega$ on $\boldsymbol{u} \in \mathcal{G}_{i Q}$ is given by

$$
\begin{aligned}
& (\omega \boldsymbol{u})_{1}=\frac{i Q}{2}+\sigma_{s}, \quad(\omega \boldsymbol{u})_{2}=\frac{i Q}{2}-\sigma_{s}, \quad(\omega \boldsymbol{u})_{3}=\theta_{1}-\theta_{\infty}, \quad(\omega \boldsymbol{u})_{4}=-\theta_{1}-\theta_{\infty}, \\
& (\omega \boldsymbol{u})_{5}=-\theta_{0}-\theta_{t}, \quad(\omega \boldsymbol{u})_{6}=\theta_{0}-\theta_{t}, \quad(\omega \boldsymbol{u})_{7}=\frac{i Q}{2}+\theta_{\infty}+\theta_{t}-\sigma_{t}, \quad(\omega \boldsymbol{u})_{8}=\frac{i Q}{2}+\theta_{\infty}+\theta_{t}+\sigma_{t} .
\end{aligned}
$$


Second, a straightforward application of the identity (B.4) yields

$$
\mathcal{B}_{h}\left(b, b^{-1}, \boldsymbol{u}\right)=\mathcal{B}_{h}\left(b, b^{-1}, \omega \boldsymbol{u}\right) \prod_{\epsilon, \epsilon^{\prime}= \pm} s_{b}\left(\epsilon \sigma_{s}+\epsilon^{\prime} \theta_{1}-\theta_{\infty}\right) s_{b}\left(\epsilon \sigma_{t}+\epsilon^{\prime} \theta_{0}+\theta_{\infty}\right)
$$

Recalling (4.5) and using $s_{b}(z)=g_{b}(z) / g_{b}(-z)$, substitution of (4.8) into (4.1) leads to

$$
F\left(b, \boldsymbol{\theta}, \sigma_{s}, \sigma_{t}\right)=X_{1} \mathcal{B}_{h}\left(b, b^{-1}, \omega \boldsymbol{u}\right),
$$

where

$$
X_{1}=\frac{1}{2} \prod_{\epsilon, \epsilon^{\prime}= \pm} \frac{g_{b}\left(\epsilon \theta_{1}+\theta_{t}+\epsilon^{\prime} \sigma_{t}\right) g_{b}\left(\epsilon \theta_{0}+\theta_{\infty}+\epsilon^{\prime} \sigma_{t}\right)}{g_{b}\left(\epsilon \theta_{0}+\theta_{t}+\epsilon^{\prime} \sigma_{s}\right) g_{b}\left(\epsilon \theta_{1}+\theta_{\infty}+\epsilon^{\prime} \sigma_{s}\right)} \prod_{\epsilon= \pm} \frac{g_{b}\left(\frac{i Q}{2}+2 \epsilon \sigma_{s}\right)}{g_{b}\left(-\frac{i Q}{2}+2 \epsilon \sigma_{t}\right)}
$$

On the other hand, using the representation (4.1) and taking $x \rightarrow x+\theta_{t}$, it can be verified that we also have

$$
F\left(b, I \hat{\boldsymbol{\theta}}, \sigma_{t}, \sigma_{s}\right)=X_{2} \mathcal{B}_{h}\left(b, b^{-1}, \omega \boldsymbol{u}\right)
$$

where $\hat{\boldsymbol{\theta}}$ is given in $(3.12), I=\operatorname{diag}(1,-1,1,1)$, and

$$
X_{2}=\frac{1}{2} \prod_{\epsilon, \epsilon^{\prime}= \pm} \frac{g_{b}\left(\epsilon \theta_{0}-\theta_{t}+\epsilon^{\prime} \sigma_{s}\right) g_{b}\left(\epsilon \theta_{1}-\theta_{\infty}+\epsilon^{\prime} \sigma_{s}\right)}{g_{b}\left(\epsilon \theta_{1}-\theta_{t}+\epsilon^{\prime} \sigma_{t}\right) g_{b}\left(\epsilon \theta_{0}-\theta_{\infty}+\epsilon^{\prime} \sigma_{t}\right)} \prod_{\epsilon= \pm} \frac{g_{b}\left(\frac{i Q}{2}+2 \epsilon \sigma_{t}\right)}{g_{b}\left(-\frac{i Q}{2}+2 \epsilon \sigma_{s}\right)}
$$

Since the Virasoro fusion kernel is even in the external momenta, we have $F\left(b, I \hat{\boldsymbol{\theta}}, \sigma_{t}, \sigma_{s}\right)=F\left(b, \hat{\boldsymbol{\theta}}, \sigma_{t}, \sigma_{s}\right)$. We deduce from (4.9) and (4.11) that the following identity holds:

$$
F\left(b, \boldsymbol{\theta}, \sigma_{s}, \sigma_{t}\right)=\frac{X_{1}}{X_{2}} F\left(b, I \hat{\boldsymbol{\theta}}, \sigma_{t}, \sigma_{s}\right),
$$

and a direct computation shows that

$$
\frac{X_{1}}{X_{2}}=\frac{\alpha\left(\boldsymbol{\theta}, \sigma_{s}\right) \alpha\left(\boldsymbol{\theta},-\sigma_{s}\right)}{\alpha\left(\hat{\boldsymbol{\theta}}, \sigma_{t}\right) \alpha\left(\hat{\boldsymbol{\theta}},-\sigma_{t}\right)}
$$

where $\alpha$ is given in (4.4). Hence, the identity (4.3) is obtained.

\subsection{Difference equations}

We now show that the Virasoro fusion kernel is a joint eigenfunction of four difference operators, the first two acting on the internal momentum $\sigma_{s}$ and the remaining two acting on its dual $\sigma_{t}$. 


\subsubsection{First pair of difference equations}

Define the difference operator $H_{F}\left(b, \boldsymbol{\theta}, \sigma_{s}\right)$ by

$$
H_{F}\left(b, \boldsymbol{\theta}, \sigma_{s}\right):=H_{F}^{+}\left(b, \boldsymbol{\theta}, \sigma_{s}\right) e^{i b \partial_{\sigma_{s}}}+H_{F}^{+}\left(b, \boldsymbol{\theta},-\sigma_{s}\right) e^{-i b \partial_{\sigma_{s}}}+H_{F}^{0}\left(b, \boldsymbol{\theta}, \sigma_{s}\right),
$$

where

$$
H_{F}^{+}\left(b, \boldsymbol{\theta}, \sigma_{s}\right)=4 \pi^{2} \frac{\Gamma\left(1+2 b^{2}-2 i b \sigma_{s}\right) \Gamma\left(b^{2}-2 i b \sigma_{s}\right) \Gamma\left(-2 i b \sigma_{s}\right) \Gamma\left(1+b^{2}-2 i b \sigma_{s}\right)}{\prod_{\epsilon, \epsilon^{\prime}= \pm 1} \Gamma\left(\frac{b Q}{2}-i b\left(\sigma_{s}+\epsilon \theta_{1}+\epsilon^{\prime} \theta_{\infty}\right)\right) \Gamma\left(\frac{b Q}{2}-i b\left(\sigma_{s}+\epsilon \theta_{0}+\epsilon^{\prime} \theta_{t}\right)\right)},
$$

and

$$
\begin{aligned}
& H_{F}^{0}\left(b, \boldsymbol{\theta}, \sigma_{s}\right)=-2 \cosh \left(2 \pi b\left(\theta_{1}+\theta_{t}+\frac{i b}{2}\right)\right) \\
& \quad+4 \sum_{k= \pm} \frac{\prod_{\epsilon= \pm} \cosh \left(\pi b\left(\epsilon \theta_{\infty}-\frac{i b}{2}-\theta_{1}-k \sigma_{s}\right)\right) \cosh \left(\pi b\left(\epsilon \theta_{0}-\frac{i b}{2}-\theta_{t}-k \sigma_{s}\right)\right)}{\sinh \left(2 \pi b\left(k \sigma_{s}+\frac{i b}{2}\right)\right) \sinh \left(2 \pi b k \sigma_{s}\right)} .
\end{aligned}
$$

In the CFT literature, the operator $H_{F}$ represents the action of a Verlinde loop operator denoted $L_{\gamma_{t}}$ on the s-channel conformal blocks (3.8). Such an operator is obtained by computing a delicate quantum monodromy operation on the s-channel blocks (3.8) along a certain closed curve $\gamma_{t}$ on the four-point Riemann sphere. The computation leading to $H_{F}$ was explicitly realized in [1, Eq. (5.37)]. It was also performed in [8, Eq. (5.31)] in a different normalization for the conformal blocks.

We now present a direct proof of the fact that the Virasoro fusion kernel (4.1) is an eigenfunction of $H_{F}$. In order to provide rigorous proofs, in the remainder of this article we will need the following assumption on the parameters:

Assumption 4.2 (Restriction on the parameters) We assume that

$$
0<b<1, \quad \boldsymbol{\theta} \in \mathbb{R}^{4}, \quad\left(\sigma_{s}, \sigma_{t}\right) \in \mathbb{R}^{2}
$$

Assumption 4.2 implies that the integration contour $\mathrm{F}$ in the Virasoro fusion kernel (4.1) is any curve going from $-\infty$ to $+\infty$ lying in the strip $\operatorname{Im} x \in]-\frac{Q}{2}, 0[$. Moreover, Assumption 4.2 is made primarily for simplicity; we expect all the results of this article to admit an analytic continuation to more general values of the parameters, such as $b \in \mathbb{C} \backslash i \mathbb{R}, \boldsymbol{\theta} \in \mathbb{C}^{4}$ and $\left(\sigma_{s}, \sigma_{t}\right) \in \mathbb{C}^{2}$.

Proposition 4.3 The Virasoro fusion kernel satisfies the following pair of difference equations:

$$
\begin{aligned}
& H_{F}\left(b, \boldsymbol{\theta}, \sigma_{s}\right) F\left(b, \boldsymbol{\theta}, \sigma_{s}, \sigma_{t}\right)=2 \cosh \left(2 \pi b \sigma_{t}\right) F\left(b, \boldsymbol{\theta}, \sigma_{s}, \sigma_{t}\right), \\
& H_{F}\left(b^{-1}, \boldsymbol{\theta}, \sigma_{s}\right) F\left(b, \boldsymbol{\theta}, \sigma_{s}, \sigma_{t}\right)=2 \cosh \left(2 \pi b^{-1} \sigma_{t}\right) F\left(b, \boldsymbol{\theta}, \sigma_{s}, \sigma_{t}\right) .
\end{aligned}
$$


Although the pair of difference Eq. (4.19) is known, we are not aware of a direct proof of it. More precisely, Eq. (4.19) are special cases of the pentagon equation which is one relation of the Moore-Seiberg groupoid [16,17,21]. They also arise from the fact that the fusion transformation (3.11) diagonalizes the Verlinde loop operator $L_{\gamma_{t}}$ [37]. We now present a direct proof of (4.19). ${ }^{2}$

Proof We only need to prove (4.19a) thanks to the symmetry (4.2) of $F$. It will be convenient to rewrite the definition (4.1) of $F$ as follows:

$$
F\left(b, \boldsymbol{\theta}, \sigma_{s}, \sigma_{t}\right)=\int_{\mathrm{F}} \mathrm{d} x X_{F}\left(x, \sigma_{s}\right) Y_{F}\left(x, \sigma_{t}\right) Z_{F}(x),
$$

where the dependence of $X_{F}\left(x, \sigma_{t}\right), Y_{F}\left(x, \sigma_{s}\right)$ and $Z_{F}(x)$ on $b$ and $\boldsymbol{\theta}$ is omitted for simplicity, and

$$
\begin{aligned}
& X_{F}\left(x, \sigma_{s}\right)=\prod_{\epsilon= \pm} \frac{g_{b}\left(\frac{i Q}{2}+2 \epsilon \sigma_{s}\right)}{s_{b}\left(x+\frac{i Q}{2}+\theta_{\infty}+\epsilon \sigma_{s}\right)} \prod_{\epsilon, \epsilon^{\prime}= \pm} g_{b}\left(\epsilon \theta_{0}+\theta_{t}+\epsilon^{\prime} \sigma_{s}\right)^{-1} g_{b}\left(\epsilon \theta_{1}-\theta_{\infty}+\epsilon^{\prime} \sigma_{s}\right)^{-1}, \\
& Y_{F}\left(x, \sigma_{t}\right)=\frac{\prod_{\epsilon, \epsilon^{\prime}= \pm} g_{b}\left(\epsilon \theta_{1}+\theta_{t}+\epsilon^{\prime} \sigma_{t}\right) g_{b}\left(\epsilon \theta_{0}-\theta_{\infty}+\epsilon^{\prime} \sigma_{t}\right)}{\prod_{\epsilon= \pm} g_{b}\left(-\frac{i Q}{2}+2 \epsilon \sigma_{t}\right) s_{b}\left(x+\frac{i Q}{2}+\theta_{t}+\epsilon \sigma_{t}\right)}, \\
& Z_{F}(x)=\prod_{\epsilon= \pm} s_{b}\left(x+\epsilon \theta_{1}\right) s_{b}\left(x+\epsilon \theta_{0}+\theta_{\infty}+\theta_{t}\right) .
\end{aligned}
$$

The proof relies on the following non-trivial identity for the action of the difference operator (4.15) on the block $X_{F}$ :

$$
\frac{H_{F}\left(b, \boldsymbol{\theta}, \sigma_{s}\right) X_{F}\left(x, \sigma_{s}\right)}{X_{F}\left(x, \sigma_{s}\right)}=\psi\left(x, \sigma_{s}\right)+2 \cosh \left(2 \pi b\left(x+\theta_{t}\right)\right),
$$

where

$\psi\left(x, \sigma_{s}\right)=-4 \prod_{\epsilon= \pm} \frac{\cosh \left(\pi b\left(x+\frac{i b}{2}+\epsilon \theta_{1}\right)\right) \cosh \left(\pi b\left(x+\frac{i b}{2}+\theta_{\infty}+\theta_{t}+\epsilon \theta_{0}\right)\right)}{\sinh \left(\pi b\left(x+i b+\theta_{\infty}+\epsilon \sigma_{s}\right)\right)}$.

Let us prove the identity (4.22). Recalling that the translation operators $e^{ \pm i b \partial_{\sigma_{s}}}$ act on $X_{F}\left(x, \sigma_{s}\right)$ as $e^{ \pm i b \partial_{\sigma_{s}}} X_{F}\left(x, \sigma_{s}\right)=X_{F}\left(x, \sigma_{s} \pm i b\right)$, the left-hand side of (4.22) takes the form

$$
\frac{H_{F}\left(b, \boldsymbol{\theta}, \sigma_{s}\right) X_{F}\left(x, \sigma_{s}\right)}{X_{F}\left(x, \sigma_{s}\right)}=H_{F}^{0}\left(b, \boldsymbol{\theta}, \sigma_{s}\right)+H_{F}^{+}\left(b, \boldsymbol{\theta}, \sigma_{s}\right) \frac{X_{F}\left(x, \sigma_{s}+i b\right)}{X_{F}\left(x, \sigma_{s}\right)}+H_{F}^{+}\left(b, \boldsymbol{\theta},-\sigma_{s}\right) \frac{X_{F}\left(x, \sigma_{s}-i b\right)}{X_{F}\left(x, \sigma_{s}\right)} .
$$

Using the identities (A.10) and (A.11) satisfied by the functions $g_{b}$ and $s_{b}$ together with the reflection equation $\Gamma(1-z) \Gamma(z)=\pi / \sin (\pi z)$ of the (Euler) Gamma function,

\footnotetext{
2 The proof is mostly inspired from the proof of [26, Theorem 3.1].
} 
we obtain

$$
\frac{H_{F}\left(b, \boldsymbol{\theta}, \sigma_{s}\right) X_{F}\left(x, \sigma_{s}\right)}{X_{F}\left(x, \sigma_{s}\right)}=H_{F}^{0}\left(b, \boldsymbol{\theta}, \sigma_{s}\right)+\phi\left(x, \sigma_{s}\right)+\phi\left(x,-\sigma_{s}\right),
$$

where $H_{F}^{0}$ is given in (4.17) and

$$
\begin{aligned}
& \phi\left(x, \sigma_{s}\right) \\
& =-\frac{4 \sinh \left(\pi b\left(x+\theta_{\infty}-\sigma_{s}\right)\right) \prod_{\epsilon= \pm} \cosh \left(\pi b\left(\epsilon \theta_{0}+\frac{i b}{2}-\theta_{t}+\sigma_{s}\right)\right) \cosh \left(\pi b\left(\epsilon \theta_{1}+\frac{i b}{2}+\theta_{\infty}+\sigma_{s}\right)\right)}{\sinh \left(2 \pi b\left(\sigma_{s}+\frac{i b}{2}\right)\right) \sinh \left(2 \pi b \sigma_{s}\right) \sinh \left(\pi b\left(x+i b+\theta_{\infty}+\sigma_{s}\right)\right)} .
\end{aligned}
$$

Hence, the proof of (4.22) is equivalent to proving the identity $f_{1}\left(\sigma_{s}\right)=f_{2}\left(\sigma_{s}\right)$ where

$$
\begin{aligned}
& f_{1}\left(\sigma_{s}\right)=\psi\left(x, \sigma_{s}\right)+2 \cosh \left(2 \pi b\left(x+\theta_{t}\right)\right), \\
& f_{2}\left(\sigma_{s}\right)=H_{F}^{0}\left(b, \boldsymbol{\theta}, \sigma_{s}\right)+\phi\left(x, \sigma_{s}\right)+\phi\left(x,-\sigma_{s}\right) .
\end{aligned}
$$

We proceed as follows: Both $f_{1}\left(\sigma_{s}\right)$ and $f_{2}\left(\sigma_{s}\right)$ are meromorphic functions of $\sigma_{s}$. It can also be easily be checked that they are even and $i b^{-1}$-periodic in $\sigma_{s}$. Moreover, we have the following asymptotics:

$$
\begin{aligned}
& \lim _{\operatorname{Re}\left(\sigma_{s}\right) \rightarrow \pm \infty} H_{F}^{0}\left(b, \boldsymbol{\theta}, \sigma_{s}\right)=0=\lim _{\operatorname{Re}\left(\sigma_{s}\right) \rightarrow \pm \infty} \psi\left(x, \sigma_{s}\right), \\
& \lim _{\operatorname{Re}\left(\sigma_{s}\right) \rightarrow \pm \infty} \phi\left(x, \sigma_{s}\right)=e^{\mp 2 \pi b\left(x+\theta_{t}\right)},
\end{aligned}
$$

which entail the asymptotics

$$
\lim _{\operatorname{Re}\left(\sigma_{s}\right) \rightarrow \pm \infty} f_{1}\left(\sigma_{s}\right)=2 \cosh \left(2 \pi b\left(x+\theta_{t}\right)\right)=\lim _{\operatorname{Re}\left(\sigma_{s}\right) \rightarrow \pm \infty} f_{2}\left(\sigma_{s}\right)
$$

We now show that $f_{1}\left(\sigma_{s}\right)$ and $f_{2}\left(\sigma_{s}\right)$ have equal $\sigma_{s}$-residues in a horizontal period strip $\operatorname{Im} \sigma_{s} \in\left[0, b^{-1}\right]$. It can first be verified that $f_{2}\left(\sigma_{s}\right)$ has vanishing residues at $\sigma_{s}= \pm \frac{i b}{2}, 0$. However, it has nonzero residues at $\sigma_{s}= \pm\left(i b+\theta_{\infty}+x\right)$ which are equal to

$$
\begin{aligned}
& \left.\operatorname{Res}\left(f_{2}\left(\sigma_{s}\right)\right)\right|_{\sigma_{s}= \pm\left(i b+\theta_{\infty}+x\right)} \\
& = \pm \frac{4}{\pi b} \sinh \left(2 \pi b\left(i b+\theta_{\infty}+x\right)\right)^{-1} \prod_{\epsilon= \pm} \cosh \left(\pi b\left(\frac{i b}{2}+x+\epsilon \theta_{1}\right)\right) \\
& \quad \cosh \left(\pi b\left(\frac{i b}{2}+\theta_{\infty}+\theta_{t}+x+\epsilon \theta_{0}\right)\right) .
\end{aligned}
$$

On the other hand, the only residues of $f_{1}\left(\sigma_{s}\right)$ are located at $\sigma_{s}= \pm\left(i b+\theta_{\infty}+x\right)$ and straightforward computations show that they are also equal to the right-hand side of (4.30). Hence, we have shown that the function $f_{1}\left(\sigma_{s}\right)-f_{2}\left(\sigma_{s}\right)$ is bounded and holomorphic in a horizontal period strip $\operatorname{Im} \sigma_{s} \in\left[0, b^{-1}\right]$. These properties extend to the whole complex plane by $i b^{-1}$-periodicity in $\sigma_{s}$. Therefore, Liouville theorem ensures that the function $f_{1}\left(\sigma_{s}\right)-f_{2}\left(\sigma_{s}\right)$ is constant everywhere. By evaluation at 
$\operatorname{Re}\left(\sigma_{s}\right)=+\infty$ using (4.29), we deduce that $f_{1}\left(\sigma_{s}\right)=f_{2}\left(\sigma_{s}\right)$. Hence, the identity (4.22) is proved.

We now let the difference operator $H_{F}$ defined in (4.15) act on the Virasoro fusion kernel (4.20). Using (4.22), we have

$$
\begin{aligned}
H_{F}\left(b, \boldsymbol{\theta}, \sigma_{s}\right) F\left(b, \boldsymbol{\theta}, \sigma_{s}, \sigma_{t}\right)= & \int_{\mathrm{F}} \mathrm{d} x X_{F}\left(x, \sigma_{s}\right) Y_{F}\left(x, \sigma_{t}\right) Z_{F}(x) \psi\left(x, \sigma_{s}\right) \\
& +2 \int_{\mathrm{F}} \mathrm{d} x X_{F}\left(x, \sigma_{s}\right) Y_{F}\left(x, \sigma_{t}\right) Z_{F}(x) \cosh \left(2 \pi b\left(x+\theta_{t}\right)\right) .
\end{aligned}
$$

The next step of the proof utilizes the following identity:

$$
\frac{Y_{F}\left(x-i b, \sigma_{t}\right)}{Y_{F}\left(x, \sigma_{t}\right)}=2 \cosh \left(2 \pi b \sigma_{t}\right)-2 \cosh \left(2 \pi b\left(x+\theta_{t}\right)\right)
$$

We now perform a contour shift together with $x \rightarrow x-i b$ in the first line of (4.31), and we use (4.32). Assumption 4.2 ensures that the contour does not cross any pole. We obtain

$$
\begin{aligned}
& H_{F}\left(b, \boldsymbol{\theta}, \sigma_{s}\right) F\left(b, \boldsymbol{\theta}, \sigma_{s}, \sigma_{t}\right)=2 \cosh \left(2 \pi b \sigma_{t}\right) \int_{\mathrm{F}} \mathrm{d} x X_{F}\left(x-i b, \sigma_{s}\right) Y_{F}\left(x, \sigma_{t}\right) \\
& Z_{F}(x-i b) \psi\left(x-i b, \sigma_{s}\right) \\
& \quad-2 \int_{\mathrm{F}} \mathrm{d} x X_{F}\left(x-i b, \sigma_{s}\right) Y_{F}\left(x, \sigma_{t}\right) Z_{F}(x-i b) \psi\left(x-i b, \sigma_{s}\right) \cosh \left(2 \pi b\left(x+\theta_{t}\right)\right) \\
& +2 \int_{\mathrm{F}} \mathrm{d} x X_{F}\left(x, \sigma_{s}\right) Y_{F}\left(x, \sigma_{t}\right) Z_{F}(x) \cosh \left(2 \pi b\left(x+\theta_{t}\right)\right) .
\end{aligned}
$$

Finally, the identity

$$
\psi\left(x-i b, \sigma_{s}\right) X_{F}\left(x-i b, \sigma_{s}\right) Z_{F}(x-i b)=X_{F}\left(x, \sigma_{s}\right) Z_{F}(x)
$$

implies that the last two lines in (4.33) cancel, and that the first line in (4.33) yields the desired result.

\subsubsection{Second pair of difference equations}

The duality transformation (4.3) exchanging the internal momenta $\sigma_{s}$ and $\sigma_{t}$ implies that the Virasoro fusion kernel (4.1) satisfies another pair of difference equations where the difference operators act on $\sigma_{t}$. We introduce the dual operator $\tilde{H}_{F}$ by

$$
\tilde{H}_{F}\left(b, \hat{\boldsymbol{\theta}}, \sigma_{t}\right):=\tilde{H}_{F}^{+}\left(b, \hat{\boldsymbol{\theta}}, \sigma_{t}\right) e^{i b \partial_{\sigma_{t}}}+\tilde{H}_{F}^{+}\left(b, \hat{\boldsymbol{\theta}},-\sigma_{t}\right) e^{-i b \partial_{\sigma_{t}}}+H_{F}^{0}\left(b, \hat{\boldsymbol{\theta}}, \sigma_{t}\right),
$$


where $H_{F}^{0}$ is given in (4.17), $\hat{\boldsymbol{\theta}}$ is defined by (3.12) and

$$
\tilde{H}_{F}^{+}\left(b, \hat{\boldsymbol{\theta}}, \sigma_{t}\right)=\frac{4 \pi^{2} \Gamma\left(1-b^{2}+2 i b \sigma_{t}\right) \Gamma\left(1+2 i b \sigma_{t}\right) \Gamma\left(2 i b \sigma_{t}-2 b^{2}\right) \Gamma\left(2 i b \sigma_{t}-b^{2}\right)}{\prod_{\epsilon, \epsilon^{\prime}= \pm} \Gamma\left(\frac{1-b^{2}}{2}+i b\left(\sigma_{t}+\epsilon \theta_{0}+\epsilon^{\prime} \theta_{\infty}\right)\right) \Gamma\left(\frac{1-b^{2}}{2}+i b\left(\sigma_{t}+\epsilon \theta_{1}+\epsilon^{\prime} \theta_{t}\right)\right)} .
$$

We next show that the Virasoro fusion kernel is also an eigenfunction of this difference operator.

Proposition 4.4 The Virasoro fusion kernel satisfies the dual pair of difference equations

$$
\begin{aligned}
& \tilde{H}_{F}\left(b, \hat{\boldsymbol{\theta}}, \sigma_{t}\right) F\left(b, \boldsymbol{\theta}, \sigma_{s}, \sigma_{t}\right)=2 \cosh \left(2 \pi b \sigma_{s}\right) F\left(b, \boldsymbol{\theta}, \sigma_{s}, \sigma_{t}\right), \\
& \tilde{H}_{F}\left(b^{-1}, \hat{\boldsymbol{\theta}}, \sigma_{t}\right) F\left(b, \boldsymbol{\theta}, \sigma_{s}, \sigma_{t}\right)=2 \cosh \left(2 \pi b^{-1} \sigma_{s}\right) F\left(b, \boldsymbol{\theta}, \sigma_{s}, \sigma_{t}\right) .
\end{aligned}
$$

Proof Again, we only need to prove (4.37a) thanks to the symmetry (4.2) of $F$. Let us first apply the duality transformation $\boldsymbol{\theta} \rightarrow \hat{\boldsymbol{\theta}}$ and $\sigma_{s} \leftrightarrow \sigma_{t}$ to Eq. (4.19a):

$$
H_{F}\left(b, \hat{\boldsymbol{\theta}}, \sigma_{t}\right) F\left(b, \hat{\boldsymbol{\theta}}, \sigma_{t}, \sigma_{s}\right)=2 \cosh \left(2 \pi b \sigma_{s}\right) F\left(b, \hat{\boldsymbol{\theta}}, \sigma_{t}, \sigma_{s}\right),
$$

where $H_{F}$ is given in (4.15). Substituting the identity (4.3) into (4.38), we obtain

$$
\left(\alpha\left(\hat{\boldsymbol{\theta}}, \pm \sigma_{t}\right)^{-1} \circ H_{F}\left(b, \hat{\boldsymbol{\theta}}, \sigma_{t}\right) \circ \alpha\left(\hat{\boldsymbol{\theta}}, \pm \sigma_{t}\right)\right) F\left(b, \boldsymbol{\theta}, \sigma_{s}, \sigma_{t}\right)=2 \cosh \left(2 \pi b \sigma_{s}\right) F\left(b, \boldsymbol{\theta}, \sigma_{s}, \sigma_{t}\right),
$$

where for the sake of brevity we denoted $f(a, \pm b)=f(a, b) f(a,-b)$. The operator on the left-hand side of (4.39) takes the form

$$
\begin{gathered}
\alpha\left(\hat{\boldsymbol{\theta}}, \pm \sigma_{t}\right)^{-1} \circ H_{F}\left(b, \hat{\boldsymbol{\theta}}, \sigma_{t}\right) \circ \alpha\left(\hat{\boldsymbol{\theta}}, \pm \sigma_{t}\right)=H_{F}^{0}\left(b, \hat{\boldsymbol{\theta}}, \sigma_{t}\right) \\
+\frac{\alpha\left(\hat{\boldsymbol{\theta}}, \sigma_{t}+i b\right)}{\alpha\left(\hat{\boldsymbol{\theta}}, \sigma_{t}\right)} \frac{\alpha\left(\hat{\boldsymbol{\theta}},-\sigma_{t}-i b\right)}{\alpha\left(\hat{\boldsymbol{\theta}},-\sigma_{t}\right)} H_{F}^{+}\left(b, \hat{\boldsymbol{\theta}}, \sigma_{t}\right) e^{i b \partial_{\sigma_{t}}} \\
+\frac{\alpha\left(\hat{\boldsymbol{\theta}}, \sigma_{t}-i b\right)}{\alpha\left(\hat{\boldsymbol{\theta}}, \sigma_{t}\right)} \frac{\alpha\left(\hat{\boldsymbol{\theta}},-\sigma_{t}+i b\right)}{\alpha\left(\hat{\boldsymbol{\theta}},-\sigma_{t}\right)} H_{F}^{+}\left(b, \hat{\boldsymbol{\theta}},-\sigma_{t}\right) \boldsymbol{e}^{-i b \partial_{\sigma_{t}}}
\end{gathered}
$$

A tedious but straightforward computation using the properties (A.10) and (A.11) of the functions $g_{b}$ and $s_{b}$ shows that for $k= \pm 1$, we have

$$
\frac{\alpha\left(\hat{\boldsymbol{\theta}}, \sigma_{t}+k i b\right)}{\alpha\left(\hat{\boldsymbol{\theta}}, \sigma_{t}\right)} \frac{\alpha\left(\hat{\boldsymbol{\theta}},-\sigma_{t}-k i b\right)}{\alpha\left(\hat{\boldsymbol{\theta}},-\sigma_{t}\right)} H_{F}^{+}\left(b, \hat{\boldsymbol{\theta}}, k \sigma_{t}\right)=\tilde{H}_{F}^{+}\left(b, \hat{\boldsymbol{\theta}}, k \sigma_{t}\right),
$$

which finally implies the identity

$$
\alpha\left(\hat{\boldsymbol{\theta}}, \pm \sigma_{t}\right)^{-1} \circ H_{F}\left(b, \hat{\boldsymbol{\theta}}, \sigma_{t}\right) \circ \alpha\left(\hat{\boldsymbol{\theta}}, \pm \sigma_{t}\right)=\tilde{H}_{F}\left(b, \hat{\boldsymbol{\theta}}, \sigma_{t}\right)
$$


In view of (4.39), this completes the proof.

In summary, we have shown that the Virasoro fusion kernel satisfies two pairs of difference equations given in (4.19) and (4.37).

\section{Main result}

The renormalized Ruijsenaars hypergeometric function (2.3) and the Virasoro fusion kernel (4.1) were studied in Sects. 2 and 4, respectively. Although they appear in different contexts, the two functions resemble each other. Both of them are proportional to a hyperbolic Barnes integral (B.2) and are joint eigenfunctions of four difference operators. The main result of this section is Theorem 1 . It shows that $F$ and $R_{\text {ren }}$ are the same function up to normalization.

The key to the identification of $F$ and $R_{\text {ren }}$ will be to compare and match the four difference equations that they satisfy. Let us first compare the difference operators $H_{F}$ defined by (4.15) and $H_{R v D}$ given in (2.9). $H_{F}$ and $H_{R v D}$ appear in the left-hand sides of the difference Eqs. (4.19a) and (2.12a) satisfied by $F$ and $R_{\text {ren }}$, respectively. Of particular importance are the "potentials" $H_{F}^{0}$ defined by (4.17) and $V$ given in (2.11), because they remain invariant under any change of normalization. The first step in the identification of $F$ and $R_{\text {ren }}$ is to show that $H_{F}^{0}$ and $V$ are equal under a certain parameter correspondence.

\subsection{Ruijsenaars/CFT parameter correspondence}

We now provide a parameter correspondence between the Virasoro fusion kernel $F\left(b, \boldsymbol{\theta}, \sigma_{s}, \sigma_{t}\right)$ and the Ruijsenaars hypergeometric function $R_{\mathrm{ren}}\left(a_{-}, a_{+}, \boldsymbol{\gamma}, v, \hat{v}\right)$. First of all, we have

$$
b=a_{-}, \quad b^{-1}=a_{+} .
$$

The second relation between the pairs $(v, \hat{v})$ and $\left(\sigma_{s}, \sigma_{t}\right)$ is simply given by

$$
v=\sigma_{s}, \quad \hat{v}=\sigma_{t}
$$

Moreover, the couplings $\boldsymbol{\gamma}$ defined by (2.1) and the external momenta $\boldsymbol{\theta}$ given in (3.7) are related by

$$
\gamma=L \theta
$$

where the matrix $L$ reads

$$
L=\left(\begin{array}{cccc}
-i & -i & 0 & 0 \\
0 & 0 & -i & i \\
i & -i & 0 & 0 \\
0 & 0 & -i & -i
\end{array}\right)
$$

Finally, the dual sets of parameters $\hat{\gamma}$ and $\hat{\boldsymbol{\theta}}$, respectively, given in (2.1) and (3.12) can be related as follows. It is straightforward to verify that the matrices $J$ defined by 
(2.2), $\mathrm{K}$ given in (3.12) and $L$ satisfy

$$
J L=L K
$$

which implies

$$
\hat{\boldsymbol{\gamma}}=L \hat{\boldsymbol{\theta}}
$$

We emphasize that the parameter correspondence described above can be rescaled thanks to the property (2.4) of the function $R_{\text {ren }}$.

It can now be verified that the potentials $H_{F}^{0}$ defined by (4.17) and $V$ given in (2.11) are equal:

$$
V\left(b, b^{-1}, L \boldsymbol{\theta}, \sigma_{s}\right)=H_{F}^{0}\left(b, \boldsymbol{\theta}, \sigma_{s}\right) .
$$

This identification suggests that the difference operator $H_{R v D}$ defined in (2.9) can be obtained from $H_{F}$ given in (4.15) after a proper change of normalization. It also suggests that the difference Eq. (4.19a) satisfied by $F$ can be mapped to the difference Eq. (2.12a) satisfied by $R_{\text {ren }}$.

\subsection{Renormalized fusion kernel $\boldsymbol{F}_{\text {ren }}$}

\subsubsection{Some requirements}

The second step in the identification of $F$ and $R_{\text {ren }}$ is to find a renormalized version $F_{\text {ren }}$ of $F$ which satisfies a difference equation of the form (2.12a), but also three other equations of the form $(2.12 b),(2.12 c),(2.12 d)$. This requirement can be reformulated as follows. The difference Eq. (2.12a) satisfied by the function $R_{\text {ren }}$ implies the three remaining Eqs. (2.12b), (2.12c) and (2.12d) because $R_{\text {ren }}$ satisfies the identities (2.5) and (2.6). Moreover, using the parameter correspondence of Sect. 5.1, the self-duality (2.6) of $R_{\text {ren }}$ becomes

$$
R_{\mathrm{ren}}\left(b, b^{-1}, L \boldsymbol{\theta}, \sigma_{s}, \sigma_{t}\right)=R_{\mathrm{ren}}\left(b, b^{-1}, L \hat{\boldsymbol{\theta}}, \sigma_{t}, \sigma_{s}\right)
$$

This equation can be compared to the property (4.3) of the Virasoro fusion kernel: The function $R_{\text {ren }}$ is self-dual, while $F$ is not. We deduce that the key to the identification of $F$ and $R_{\text {ren }}$ is to find a renormalized version $F_{\text {ren }}$ of $F$ which (i) satisfies a difference equation of the type (2.12a), (ii) is invariant under $b \rightarrow b^{-1}$, and (iii) is self-dual.

\subsubsection{Definition of $F_{\text {ren }}$}

We now present a renormalized version $F_{\text {ren }}$ of $F$ which satisfies the three properties (i)-(iii). First, we define renormalized conformal blocks $\mathcal{F}_{\text {ren }}$ by

$$
\mathcal{F}_{\text {ren }}\left(b, \boldsymbol{\theta}, \sigma_{s} ; z\right)=N\left(\theta_{\infty}, \theta_{1}, \sigma_{s}\right) N\left(\sigma_{s}, \theta_{t}, \theta_{0}\right) \mathcal{F}\left(b, \boldsymbol{\theta}, \sigma_{s} ; z\right),
$$


where $\mathcal{F}$ is given in (3.8), and where the normalization factor $N$ is

$$
N\left(\theta_{3}, \theta_{2}, \theta_{1}\right)=\frac{g_{b}\left(-\theta_{1}-\theta_{2}-\theta_{3}\right) g_{b}\left(\theta_{1}-\theta_{2}-\theta_{3}\right) g_{b}\left(-\theta_{1}-\theta_{2}+\theta_{3}\right) g_{b}\left(\theta_{1}-\theta_{2}+\theta_{3}\right)}{g_{b}\left(-2 \theta_{1}+\frac{i Q}{2}\right) g_{b}\left(-2 \theta_{2}+\frac{i Q}{2}\right) g_{b}\left(2 \theta_{3}+\frac{i Q}{2}\right)} .
$$

We are now going to rewrite the fusion transformation (3.11) in terms of $\mathcal{F}_{\text {ren }}$. We first introduce a weight function ${ }^{3}$

$$
\omega\left(b, \boldsymbol{\theta}, \sigma_{s}\right)=\mu\left(b, \boldsymbol{\theta}, \sigma_{s}\right) \mu\left(b, \boldsymbol{\theta},-\sigma_{s}\right),
$$

where

$$
\mu\left(b, \boldsymbol{\theta}, \sigma_{s}\right)=\frac{s_{b}\left(\frac{i Q}{2}+2 \sigma_{s}\right)}{\prod_{\epsilon= \pm 1} s_{b}\left(\sigma_{s}-\theta_{1}+\epsilon \theta_{\infty}\right) s_{b}\left(\sigma_{s}-\theta_{t}+\epsilon \theta_{0}\right)} .
$$

The fusion transformation (3.11) can now be rewritten as

$$
\mathcal{F}_{\text {ren }}\left(b, \boldsymbol{\theta}, \sigma_{s} ; z\right)=\int_{\mathbb{R}_{+}} d \sigma_{t} \omega\left(b, \hat{\boldsymbol{\theta}}, \sigma_{t}\right) F_{\text {ren }}\left(b, \boldsymbol{\theta}, \sigma_{s}, \sigma_{t}\right) \mathcal{F}_{\text {ren }}\left(b, \hat{\boldsymbol{\theta}}, \sigma_{t} ; 1-z\right),
$$

where the renormalized Virasoro fusion kernel $F_{\text {ren }}$ is defined by

$$
F_{\text {ren }}\left(b, \boldsymbol{\theta}, \sigma_{s}, \sigma_{t}\right)=\omega\left(b, \hat{\boldsymbol{\theta}}, \sigma_{t}\right)^{-1} \frac{N\left(\theta_{\infty}, \theta_{1}, \sigma_{s}\right) N\left(\sigma_{s}, \theta_{t}, \theta_{0}\right)}{N\left(\theta_{\infty}, \theta_{0}, \sigma_{t}\right) N\left(\sigma_{t}, \theta_{t}, \theta_{1}\right)} F\left(b, \boldsymbol{\theta}, \sigma_{s}, \sigma_{t}\right),
$$

and where $F$ is defined in (4.1). In the next three propositions, we show that $F_{\text {ren }}$ satisfies the desired three properties (i)-(iii).

Proposition 5.1 The following identity holds:

$$
F_{\text {ren }}\left(b^{-1}, \boldsymbol{\theta}, \sigma_{s}, \sigma_{t}\right)=F_{\text {ren }}\left(b, \boldsymbol{\theta}, \sigma_{s}, \sigma_{t}\right) .
$$

Proof Equation (5.15) follows from the symmetries $s_{b}(x)=s_{b^{-1}}(x)$ and $g_{b}(x)=$ $g_{b^{-1}}(x)$.

We now prove that $F_{\text {ren }}$ is self-dual.

Proposition 5.2 The renormalized Virasoro fusion kernel $F_{\text {ren }}$ satisfies

$$
F_{r e n}\left(b, \boldsymbol{\theta}, \sigma_{s}, \sigma_{t}\right)=F_{r e n}\left(b, \hat{\boldsymbol{\theta}}, \sigma_{t}, \sigma_{s}\right)
$$

\footnotetext{
3 The weight function $\omega$ was introduced by Ruijsenaars in [28, Eq. (1.15)] in order to construct a unitary eigenfunction transform associated with the function $R_{\text {ren }}$.
} 
Proof The proof consists of writing an explicit relation between $F_{\text {ren }}\left(b, \boldsymbol{\theta}, \sigma_{s}, \sigma_{t}\right)$ and $F_{\text {ren }}\left(b, \hat{\boldsymbol{\theta}}, \sigma_{t}, \sigma_{s}\right)$ using the definition (5.14) and the identity (4.3). First, from (5.14) we have

$$
F_{\text {ren }}\left(b, \hat{\boldsymbol{\theta}}, \sigma_{t}, \sigma_{s}\right)=\omega\left(b, \boldsymbol{\theta}, \sigma_{s}\right)^{-1} \frac{N\left(\theta_{\infty}, \theta_{0}, \sigma_{t}\right) N\left(\sigma_{t}, \theta_{t}, \theta_{1}\right)}{N\left(\theta_{\infty}, \theta_{1}, \sigma_{s}\right) N\left(\sigma_{s}, \theta_{t}, \theta_{0}\right)} F\left(b, \hat{\boldsymbol{\theta}}, \sigma_{t}, \sigma_{s}\right) .
$$

Substituting the identity (4.3) into (5.17), we have

$$
F_{\mathrm{ren}}\left(b, \hat{\boldsymbol{\theta}}, \sigma_{t}, \sigma_{s}\right)=\omega\left(b, \boldsymbol{\theta}, \sigma_{s}\right)^{-1} \frac{N\left(\theta_{\infty}, \theta_{0}, \sigma_{t}\right) N\left(\sigma_{t}, \theta_{t}, \theta_{1}\right)}{N\left(\theta_{\infty}, \theta_{1}, \sigma_{s}\right) N\left(\sigma_{s}, \theta_{t}, \theta_{0}\right)} \frac{\alpha\left(\hat{\boldsymbol{\theta}}, \sigma_{t}\right) \alpha\left(\hat{\boldsymbol{\theta}},-\sigma_{t}\right)}{\alpha\left(\boldsymbol{\theta}, \sigma_{s}\right) \alpha\left(\boldsymbol{\theta},-\sigma_{s}\right)} F\left(b, \boldsymbol{\theta}, \sigma_{s}, \sigma_{t}\right),
$$

where $\alpha$ is given in (4.4). Using once again the definition (5.14), we obtain

$$
\begin{aligned}
& F_{\text {ren }}\left(b, \hat{\boldsymbol{\theta}}, \sigma_{t}, \sigma_{s}\right)=\omega\left(b, \boldsymbol{\theta}, \sigma_{s}\right)^{-1} \frac{N\left(\theta_{\infty}, \theta_{0}, \sigma_{t}\right) N\left(\sigma_{t}, \theta_{t}, \theta_{1}\right)}{N\left(\theta_{\infty}, \theta_{1}, \sigma_{s}\right) N\left(\sigma_{s}, \theta_{t}, \theta_{0}\right)} \\
& \quad \times \frac{\alpha\left(\hat{\boldsymbol{\theta}}, \sigma_{t}\right) \alpha\left(\hat{\boldsymbol{\theta}},-\sigma_{t}\right)}{\alpha\left(\boldsymbol{\theta}, \sigma_{s}\right) \alpha\left(\boldsymbol{\theta},-\sigma_{s}\right)} \omega\left(b, \hat{\boldsymbol{\theta}}, \sigma_{t}\right) \frac{N\left(\theta_{\infty}, \theta_{0}, \sigma_{t}\right) N\left(\sigma_{t}, \theta_{t}, \theta_{1}\right)}{N\left(\theta_{\infty}, \theta_{1}, \sigma_{s}\right) N\left(\sigma_{s}, \theta_{t}, \theta_{0}\right)} F_{\mathrm{ren}}\left(b, \boldsymbol{\theta}, \sigma_{s}, \sigma_{t}\right) .
\end{aligned}
$$

Finally, a direct computation using $s_{b}(x)=g_{b}(x) / g_{b}(-x)$ shows that all the prefactors in the right-hand side of (5.19) cancel out. Therefore, the identity (5.16) is obtained.

\subsection{Difference equations for $F_{\text {ren }}$}

We now show that the renormalized Virasoro fusion kernel and the renormalized Ruijsenaars hypergeometric function satisfy the same four difference equations.

Proposition 5.3 The renormalized fusion kernel $F_{\text {ren }}$ defined by (5.14) satisfies the following difference equations:

$$
\begin{aligned}
& H_{R v D}\left(b, b^{-1}, L \boldsymbol{\theta}, \sigma_{s}\right) F_{r e n}\left(b, \boldsymbol{\theta}, \sigma_{s}, \sigma_{t}\right)=2 \cosh \left(2 \pi b \sigma_{t}\right) F_{r e n}\left(b, \boldsymbol{\theta}, \sigma_{s}, \sigma_{t}\right), \\
& H_{R v D}\left(b^{-1}, b, L \boldsymbol{\theta}, \sigma_{s}\right) F_{r e n}\left(b, \boldsymbol{\theta}, \sigma_{s}, \sigma_{t}\right)=2 \cosh \left(2 \pi b^{-1} \sigma_{t}\right) F_{r e n}\left(b, \boldsymbol{\theta}, \sigma_{s}, \sigma_{t}\right), \\
& H_{R v D}\left(b, b^{-1}, L \hat{\boldsymbol{\theta}}, \sigma_{t}\right) F_{r e n}\left(b, \boldsymbol{\theta}, \sigma_{s}, \sigma_{t}\right)=2 \cosh \left(2 \pi b \sigma_{s}\right) F_{r e n}\left(b, \boldsymbol{\theta}, \sigma_{s}, \sigma_{t}\right), \\
& H_{R v D}\left(b^{-1}, b, L \hat{\boldsymbol{\theta}}, \sigma_{t}\right) F_{r e n}\left(b, \boldsymbol{\theta}, \sigma_{s}, \sigma_{t}\right)=2 \cosh \left(2 \pi b^{-1} \sigma_{s}\right) F_{r e n}\left(b, \boldsymbol{\theta}, \sigma_{s}, \sigma_{t}\right),
\end{aligned}
$$

where the matrix $L$ is given in (5.4) and $H_{R v D}$ is defined by (2.9).

Proof We only need to prove (5.20a) thanks to the symmetries (5.15) and (5.16) of $F_{\text {ren. }}$ Moreover, it will be convenient to rewrite (5.14) as $F=\left(P\left(\sigma_{t}\right) / Q\left(\sigma_{s}\right)\right) F_{\text {ren }}$, 
where

$$
\begin{aligned}
& P\left(\sigma_{t}\right)=\omega\left(b, \hat{\boldsymbol{\theta}}, \sigma_{t}\right) N\left(\theta_{\infty}, \theta_{0}, \sigma_{t}\right) N\left(\sigma_{t}, \theta_{t}, \theta_{1}\right), \\
& Q\left(\sigma_{s}\right)=N\left(\theta_{\infty}, \theta_{1}, \sigma_{s}\right) N\left(\sigma_{s}, \theta_{t}, \theta_{0}\right) .
\end{aligned}
$$

Substituting $F=\left(P\left(\sigma_{t}\right) / Q\left(\sigma_{s}\right)\right) F_{\text {ren }}$ into the difference Eq. (4.19a) satisfied by $F$, we obtain

$$
\left(\frac{Q\left(\sigma_{s}\right)}{P\left(\sigma_{t}\right)} \circ H_{F}\left(b, \boldsymbol{\theta}, \sigma_{S}\right) \circ \frac{P\left(\sigma_{t}\right)}{Q\left(\sigma_{s}\right)}\right) F_{\mathrm{ren}}\left(b, \boldsymbol{\theta}, \sigma_{s}, \sigma_{t}\right)=2 \cosh \left(2 \pi b \sigma_{t}\right) F_{\mathrm{ren}}\left(b, \boldsymbol{\theta}, \sigma_{s}, \sigma_{t}\right)
$$

The factors $P\left(\sigma_{t}\right)$ in the left-hand side of (5.22) cancel out, since the difference operator $H_{F}\left(b, \boldsymbol{\theta}, \sigma_{s}\right)$ acts on the variable $\sigma_{s}$. Therefore, the proof of (5.20a) consists of verifying the following identity:

$$
Q\left(\sigma_{s}\right) \circ H_{F}\left(b, \boldsymbol{\theta}, \sigma_{s}\right) \circ Q\left(\sigma_{s}\right)^{-1}=H_{R v D}\left(b, b^{-1}, L \boldsymbol{\theta}, \sigma_{s}\right),
$$

where $H_{F}$ and $H_{R v D}$ are, respectively, defined by (4.15) and (2.9). We have

$$
\begin{aligned}
& Q\left(\sigma_{s}\right) \circ H_{F}\left(b, \boldsymbol{\theta}, \sigma_{s}\right) \circ Q\left(\sigma_{s}\right)^{-1} \\
& \quad=H_{F}^{0}\left(b, \boldsymbol{\theta}, \sigma_{s}\right)+\frac{Q\left(\sigma_{s}\right)}{Q\left(\sigma_{s}+i b\right)} H_{F}^{+}\left(b, \boldsymbol{\theta}, \sigma_{s}\right) e^{i b \partial_{\sigma_{s}}}+\frac{Q\left(\sigma_{s}\right)}{Q\left(\sigma_{s}-i b\right)} H_{F}^{+}\left(b, \boldsymbol{\theta},-\sigma_{s}\right) e^{-i b \partial_{\sigma_{s}}},
\end{aligned}
$$

where $H_{F}^{+}$is given in (4.16). Using the identity (A.10) satisfied by the $g_{b}$-function, it is straightforward to verify that the following identities hold for $k= \pm 1$ :

$$
\begin{aligned}
\frac{Q\left(\sigma_{s}\right)}{Q\left(\sigma_{s}+k i b\right)}= & \frac{\Gamma\left(1-b^{2}+2 i b k \sigma_{s}\right) \Gamma\left(1+2 i b k \sigma_{s}\right)}{\Gamma\left(1+b^{2}-2 i b k \sigma_{s}\right) \Gamma\left(1+2 b^{2}-2 i b k \sigma_{s}\right)} \\
& \times \prod_{\epsilon= \pm} \frac{\Gamma\left(\frac{b Q}{2}+i b\left(\epsilon \theta_{0}+\theta_{t}-k \sigma_{s}\right)\right) \Gamma\left(\frac{b Q}{2}+i b\left(\epsilon \theta_{\infty}+\theta_{1}-k \sigma_{s}\right)\right)}{\left.\frac{b Q}{2}+i b\left(\epsilon \theta_{0}+\theta_{t}+k \sigma_{s}\right)\right) \Gamma\left(1-\frac{b Q}{2}+i b\left(\epsilon \theta_{\infty}+\theta_{1}+k \sigma_{s}\right)\right)}
\end{aligned}
$$

We now substitute (5.25) into (5.24), and we use the reflection equation $\Gamma(1-z) \Gamma(z)=$ $\pi / \sin (\pi z)$. For $k= \pm 1$, we obtain

$$
\frac{Q\left(\sigma_{s}\right)}{Q\left(\sigma_{s}+k i b\right)} H_{F}^{+}\left(b, \boldsymbol{\theta}, k \sigma_{s}\right)=C\left(b, b^{-1}, L \boldsymbol{\theta},-k \sigma_{s}\right)
$$

where $C$ is defined by (2.10). It finally remains to use the identification (5.7) of the potentials $V$ and $H_{F}^{0}$ to obtain (5.23).

\section{$5.4 F_{\text {ren }}=R_{\text {ren }}$}

We have shown that the renormalized Virasoro fusion kernel (5.14) and the renormalized Ruijsenaars hypergeometric function (2.3) satisfy the same four difference 
equations. Therefore, the two functions are proportional. We now present the main result of this article.

Theorem 1 The renormalized fusion kernel $F_{\text {ren }}$ given in (5.14) and the renormalized Ruijsenaars hypergeometric function defined by (2.3) are equal:

$$
F_{\text {ren }}\left(b, \boldsymbol{\theta}, \sigma_{s}, \sigma_{t}\right)=R_{\text {ren }}\left(b, b^{-1}, L \boldsymbol{\theta}, \sigma_{s}, \sigma_{t}\right),
$$

where the matrix $L$ is given in (5.4).

Proof The equality is not immediate and follows from the identity (B.4) satisfied by the hyperbolic Barnes integral. Let us first rewrite $F_{\text {ren }}$ using (5.14) and the representation (4.1). It will also be convenient to perform a shift $x \rightarrow x-\frac{i Q}{2}$ in the integrand. This amounts to lifting up all poles of the integrand by $\frac{i Q}{2}$. Because of Assumption 4.2, this shift maps the contour $F$ to a contour $F^{\prime}$ which runs from $-\infty$ and $+\infty$ and lies in the strip $\operatorname{Im} x \in] 0, \frac{Q}{2}$ [. It is now straightforward to verify that $F_{\text {ren }}$ can be written as

$$
\begin{aligned}
F_{\text {ren }}\left(b, \boldsymbol{\theta}, \sigma_{s}, \sigma_{t}\right)= & \prod_{\epsilon_{1}= \pm}\left(\frac{s_{b}\left(\epsilon_{1} \sigma_{t}-\theta_{0}-\theta_{\infty}\right)}{s_{b}\left(\epsilon_{1} \sigma_{s}+\theta_{1}-\theta_{\infty}\right)} \prod_{\epsilon_{2}= \pm} s_{b}\left(\epsilon_{1} \sigma_{s}+\epsilon_{2} \theta_{0}-\theta_{t}\right)\right) \\
& \times \int_{\mathrm{F}^{\prime}} \mathrm{d} x \prod_{\epsilon= \pm} \frac{s_{b}\left(x-\frac{i Q}{2}+\epsilon \theta_{1}\right) s_{b}\left(x-\frac{i Q}{2}+\theta_{\infty}+\theta_{t}+\epsilon \theta_{0}\right)}{s_{b}\left(x+\theta_{\infty}+\epsilon \sigma_{s}\right) s_{b}\left(x+\theta_{t}+\epsilon \sigma_{t}\right)} .
\end{aligned}
$$

On the other hand, the function $R_{\text {ren }}$ defined in (2.3) satisfies

$$
\begin{aligned}
& R_{\mathrm{ren}}\left(b, b^{-1}, L \boldsymbol{\theta}, \sigma_{s}, \sigma_{t}\right)=\prod_{\epsilon= \pm 1} \frac{s_{b}\left(\epsilon \sigma_{s}-\theta_{0}-\theta_{t}\right)}{s_{b}\left(\epsilon \sigma_{t}+\theta_{1}+\theta_{t}\right)} \\
& \quad \times \int_{\mathrm{R}} d z \frac{1}{s_{b}\left(z+\frac{i Q}{2}\right) s_{b}\left(z+2 \theta_{t}+\frac{i Q}{2}\right)} \prod_{\epsilon= \pm 1} \frac{s_{b}\left(z+\theta_{0}+\theta_{t}+\epsilon \sigma_{s}\right) s_{b}\left(z+\theta_{1}+\theta_{t}+\epsilon \sigma_{t}\right)}{s_{b}\left(z+\theta_{0}+\theta_{1}+\epsilon \theta_{\infty}+\theta_{t}+\frac{i Q}{2}\right)},
\end{aligned}
$$

where the contour R runs from $-\infty$ to $+\infty$ lying in the strip $\operatorname{Im} z \in]-\frac{Q}{2}, 0[$. Therefore, the proof of Theorem 1 consists of proving that the functions (5.28) and (5.29) are equal. Recalling the definition (B.2) of the hyperbolic Barnes integral and using the identity $s_{b}(z)=G\left(b, b^{-1}, z\right)$, the contour integral in (5.28) takes the form

$$
\int_{\mathrm{F}^{\prime}} \mathrm{d} x \prod_{\epsilon= \pm} \frac{s_{b}\left(x-\frac{i Q}{2}+\epsilon \theta_{1}\right) s_{b}\left(x-\frac{i Q}{2}+\theta_{\infty}+\theta_{t}+\epsilon \theta_{0}\right)}{s_{b}\left(x+\theta_{\infty}+\epsilon \sigma_{s}\right) s_{b}\left(x+\theta_{t}+\epsilon \sigma_{t}\right)}=\frac{1}{2} \mathcal{B}_{h}\left(b, b^{-1}, \boldsymbol{v}\right) .
$$

Several choices of $\boldsymbol{v} \in \mathcal{G}_{i}$ lead to the same contour integral. Here, we choose

$$
\begin{aligned}
& v_{1}=\theta_{t}-\sigma_{t}, \quad v_{2}=\theta_{t}+\sigma_{t}, \quad v_{3}=\frac{i Q}{2}+\theta_{1}, \quad v_{4}=\frac{i Q}{2}-\theta_{0}-\theta_{\infty}-\theta_{t}, \\
& v_{5}=\frac{i Q}{2}-\theta_{1}, \quad v_{6}=\frac{i Q}{2}+\theta_{0}-\theta_{\infty}-\theta_{t}, \quad v_{7}=\theta_{\infty}-\sigma_{s}, \quad v_{8}=\theta_{\infty}+\sigma_{s} .
\end{aligned}
$$


From (B.3), we have $s=\frac{\theta_{0}-\theta_{1}+\theta_{\infty}-\theta_{t}}{2}$, and the action of $\omega$ on the parameters $\boldsymbol{v} \in \mathcal{G}_{i} Q$ yields

$$
\omega \boldsymbol{v}=\left(v_{1}+s, v_{2}+s, \ldots, v_{5}-s, \ldots, v_{8}-s\right) .
$$

Finally, the equality between the functions (5.28) and (5.29) is found after applying the identity (B.4) on (5.30), performing the change of variable $x=-z-\frac{\theta_{1}}{2}-\frac{\theta_{0}}{2}-\frac{\theta_{\infty}}{2}-\frac{3 \theta_{t}}{2}$ which maps the contour $\mathrm{F}^{\prime}$ to $\mathrm{R}$, and using the identity $s_{b}(z)=s_{b}(-z)^{-1}$.

\section{Conclusion and perspectives}

In this article, we have proved that the Virasoro fusion kernel is a joint eigenfunction of four difference operators. We have found a normalization of the conformal blocks for which the four difference operators are mapped to four versions of the quantum relativistic hyperbolic $B C_{1}$ Calogero-Moser Hamiltonian. We have consequently proved that the Virasoro fusion kernel and the Ruijsenaars hypergeometric function coincide up to normalization and are the quantum eigenfunction of this integrable system. We now mention a list of perspectives related to this work.

(1) It would be interesting to understand the role played by the four-point Virasoro conformal blocks in the context of the present integrable system. In view of (5.13) and Theorem 1, the function $R_{\text {ren }}$ is the kernel of the fusion transformation relating the $s$ - and $t$-channel renormalized conformal blocks. On the other hand, in [28] a unitary Hilbert space transform associated with the function $R_{\text {ren }}$ was constructed for special values of the couplings. We believe that this Hilbert space is in fact spanned by the four-point Virasoro conformal blocks.

(2) The quantum eigenfunction of the $A_{1}$ relativistic hyperbolic CM system is a onecoupling specialization of the Ruijsenaars hypergeometric function [29]. It would be interesting to compare this limit to the transition limit from the Virasoro fusion kernel to the Virasoro modular kernel [11]. The latter describes how conformal blocks associated with the one-point torus transform under a mapping class group action [18].

(3) A natural question is to find higher rank generalizations of our result. The $B C_{N}$, $N>1$ generalization of the function $R_{\text {ren }}$ has not yet been constructed. Higher rank analogs of the Virasoro fusion kernel are associated with $\mathcal{W}$-type algebras and also remain to be found. However, the framework developed in [33,34] allows us, in principle, to construct such a generalization from a quantum group perspective.

(4) What is the meaning of the classical and/or non-relativistic limits in the conformal blocks setting?

(5) The $R$-function reduces to the celebrated Askey-Wilson polynomials in a limit where one of $v, \hat{v}$ is discretized [26]. What does this limit mean from the conformal blocks viewpoint?

(6) The Askey-Wilson polynomials can be studied using representation theory of the double affine Hecke algebra and the Askey-Wilson algebra [14,19]. An interest- 
ing program would be to generalize this algebraic study to the case of $R_{\text {ren }}$. We believe that Virasoro conformal blocks and their quantum monodromies provide the correct framework for such a study.

(7) Various confluent limits of the Virasoro fusion kernel were constructed in [15]. It would be interesting to understand these limits from an integrable system point of view.

(8) An interesting project motivated by the WZW $S L(2, R)$ model would be to construct the fusion kernel of the affine Lie algebra $\hat{s l}_{2}$ and to find a connection to integrable systems.

(9) Due to the role played by the Virasoro fusion kernel in the bootstrap approach to Liouville theory [21,23], we hope that the present work can provide new insights on the role of integrability in two-dimensional CFTs.

Acknowledgements The author would like to thank P. Baseilhac, E. Langmann, S. Ribault and S. Ruijsenaars for valuable discussions and encouragements. The author is also grateful to O. Lisovyy for many helpful discussions and for his comments on a first version of the manuscript. Finally, the author is indebted to J. Lenells for his encouragements and his careful checks on the latest version of this paper. Support is acknowledged from the European Research Council, Grant Agreement No. 682537. The author states that there is no conflict of interest.

Funding Open Access funding provided by Royal Institute of Technology

Open Access This article is licensed under a Creative Commons Attribution 4.0 International License, which permits use, sharing, adaptation, distribution and reproduction in any medium or format, as long as you give appropriate credit to the original author(s) and the source, provide a link to the Creative Commons licence, and indicate if changes were made. The images or other third party material in this article are included in the article's Creative Commons licence, unless indicated otherwise in a credit line to the material. If material is not included in the article's Creative Commons licence and your intended use is not permitted by statutory regulation or exceeds the permitted use, you will need to obtain permission directly from the copyright holder. To view a copy of this licence, visit http://creativecommons.org/licenses/by/4.0/.

\section{Appendix A. Special functions}

Equation (2.3) expresses the renormalized Ruijsenaars hypergeometric function in terms of the hyperbolic gamma function $G\left(a_{-}, a_{+}, z\right)=E\left(a_{-}, a_{+}, z\right) / E\left(a_{-}, a_{+},-z\right)$. Let us omit the dependence of $G$ and $E$ on $a_{-}$and $a_{+}$for simplicity. Following [26], the two functions are defined by

$$
G(z)=\exp \left[i \int_{0}^{\infty} \frac{d y}{y}\left(\frac{\sin 2 y z}{2 \sinh \left(a_{+} y\right) \sinh \left(a_{-} y\right)}-\frac{z}{a_{+} a_{-} y}\right)\right], \quad|\operatorname{Im} z|<a,
$$

and

$$
E(z)=\exp \left[\frac{1}{4} \int_{0}^{\infty} \frac{d y}{y}\left(\frac{1-e^{-2 i y z}}{\sinh \left(a_{+} y\right) \sinh \left(a_{-} y\right)}-\frac{2 i z}{a_{+} a_{-} y}-\frac{z^{2}}{a_{+} a_{-}}\left(e^{-2 a_{+} y}+e^{-2 a_{-} y}\right)\right)\right], \quad \operatorname{Im} z<\operatorname{Re} a,
$$


where $a=\left(a_{+}+a_{-}\right) / 2$. The functions $G$ and $E$ are obviously invariant under the exchange of $a_{-}$and $a_{+}$. Moreover, $G$ satisfies the difference equations

$$
\frac{G\left(z+i a_{-} / 2\right)}{G\left(z-i a_{-} / 2\right)}=2 \cosh \left(\pi z / a_{+}\right), \quad \frac{G\left(z+i a_{+} / 2\right)}{G\left(z-i a_{+} / 2\right)}=2 \cosh \left(\pi z / a_{-}\right) .
$$

As long as the ratio $a_{-} / a_{+}$stays in the subset $\mathbb{C} \backslash(-\infty, 0]$, the function $G$ extends to a meromorphic function with poles and zeros given by

$$
\begin{gathered}
z=-i\left(k+\frac{1}{2}\right) a_{-}-i\left(l+\frac{1}{2}\right) a_{+}, \quad k, l=0,1,2, \ldots, \quad \text { (poles) } \\
z=i\left(k+\frac{1}{2}\right) a_{-}+i\left(l+\frac{1}{2}\right) a_{+}, \quad k, l=0,1,2, \ldots, \quad \text { (zeros) }
\end{gathered}
$$

Finally, the function $G$ is scale invariant:

$$
G\left(\lambda a_{-}, \lambda a_{+}, \lambda z\right)=G\left(a_{-}, a_{,} z\right), \quad \lambda>0 .
$$

The function $E$ satisfies the difference equation

$$
\frac{E\left(z+i a_{-} / 2\right)}{E\left(z-i a_{-} / 2\right)}=\frac{\sqrt{2 \pi}}{\Gamma\left(\frac{1}{2}+\frac{i z}{a_{+}}\right)}\left(\frac{a_{-}}{a_{+}}\right)^{\frac{i z}{2 a_{+}}}
$$

and the difference equation obtained by exchanging $a_{-}$and $a_{+}$in (A.7). The function $E$ is holomorphic as long as $a_{-} / a_{+} \in \mathbb{C} \backslash(-\infty, 0]$. Moreover, $E$ has zeros located at

$$
z=i\left(k+\frac{1}{2}\right) a_{-}+i\left(l+\frac{1}{2}\right) a_{+}, \quad k, l=0,1,2, \ldots
$$

On the other hand, the Virasoro fusion kernel (4.1) is defined in terms of two special functions $s_{b}(z)$ and $g_{b}(z)$ such that $s_{b}(z)=g_{b}(z) / g_{b}(-z)$. They are related to the functions $G$ and $E$ as follows:

$$
s_{b}(z)=G\left(b, b^{-1} ; z\right), \quad g_{b}(z)=\frac{1}{E\left(b, b^{-1} ;-z\right)} .
$$

The properties of $s_{b}$ and $g_{b}$ can be deduced from the ones of $G$ and $E$. Both $s_{b}$ and $g_{b}$ are obviously invariant under the exchange of $b$ and $b^{-1}$. Most importantly, the function $g_{b}(z)$ satisfies the difference equations

$$
\frac{g_{b}\left(z+\frac{i b}{2}\right)}{g_{b}\left(z-\frac{i b}{2}\right)}=\frac{b^{-i b z} \sqrt{2 \pi}}{\Gamma\left(\frac{1}{2}-i b z\right)}, \quad \frac{g_{b}\left(z+\frac{i}{2 b}\right)}{g_{b}\left(z-\frac{i}{2 b}\right)}=\frac{b^{-\frac{i z}{b}} \sqrt{2 \pi}}{\Gamma\left(\frac{1}{2}-\frac{i z}{b}\right)} .
$$

Finally, the $s_{b}$ function satisfies

$$
\frac{s_{b}\left(z+\frac{i b}{2}\right)}{s_{b}\left(z-\frac{i b}{2}\right)}=2 \cosh \pi b z, \quad \frac{s_{b}\left(z+\frac{i}{2 b}\right)}{s_{b}\left(z-\frac{i}{2 b}\right)}=2 \cosh \frac{\pi z}{b}
$$




\section{Appendix B. Hyperbolic Barnes integral}

In this appendix, we define the hyperbolic Barnes integral $\mathcal{B}_{h}\left(a_{-}, a_{+}, \boldsymbol{u}\right)$ following [5]. We describe one of its symmetry properties which is the key to the proofs of Proposition 4.1 and Theorem 1.

Define the complex hyperplane $\mathcal{G}_{k}$ such that

$$
\mathcal{G}_{k}=\left\{u=\left(u_{1}, \ldots u_{8}\right) \in \mathbb{C}^{8} \mid \sum_{j=1}^{8} u_{j}=2 k\right\} .
$$

The hyperbolic Barnes integral $\mathcal{B}_{h}\left(a_{-}, a_{+}, \boldsymbol{u}\right)$ is defined for $\boldsymbol{u} \in \mathcal{G}_{2 i a}$ and $a=\frac{a_{-}+a_{+}}{2}$ by

$$
\mathcal{B}_{h}\left(a_{-}, a_{+}, \boldsymbol{u}\right)=2 \int_{\mathcal{C}} \frac{\prod_{j=3}^{6} G\left(z-u_{j}\right)}{\prod_{j=1,2,7,8}^{6} G\left(z+u_{j}\right)} d z,
$$

where the contour $\mathcal{C}$ goes from $-\infty$ and $+\infty$, passing in between the upper and lower sequences of poles of the integrand.

Lemma B.1 [5, Proposition 4.7] Let $\omega$ be an operator acting on $\boldsymbol{u} \in \mathcal{G}_{2 i a}$ as

$$
\omega \boldsymbol{u}=\left(u_{1}+s, \ldots, u_{4}+s, u_{5}-s, \ldots, u_{8}-s\right), \quad s=i a-\frac{1}{2}\left(u_{1}+u_{2}+u_{3}+u_{4}\right) .
$$

The hyperbolic Barnes integral $B_{h}\left(a_{-}, a_{+}, \boldsymbol{u}\right), \boldsymbol{u} \in \mathcal{G}_{2 i a}$ is invariant under permutations of $\left(u_{1}, u_{2}, u_{7}, u_{8}\right)$ and of $\left(u_{3}, u_{4}, u_{5}, u_{6}\right)$, and it satisfies ${ }^{4}$

$$
\mathcal{B}_{h}\left(a_{-}, a_{+}, \boldsymbol{u}\right)=\mathcal{B}_{h}\left(a_{-}, a_{+}, \omega \boldsymbol{u}\right) \prod_{j=1,2} \prod_{k=3,4} G\left(i a-u_{j}-u_{k}\right) \prod_{j=5,6} \prod_{k=7,8} G\left(i a-u_{j}-u_{k}\right) .
$$

\section{References}

1. Alday, L.F., Gaiotto, D., Gukov, S., Tachikawa, Y., Verlinde, H.: Loop and surface operators in N=2 gauge theory and Liouville modular geometry. J. High Energy Phys. 2010, 113 (2010)

2. Alday, L.F., Gaiotto, D., Tachikawa, Y.: Liouville correlation functions from four-dimensional gauge theories. Lett. Math. Phys. 91, 167-197 (2010)

3. Belavin, A.A., Polyakov, A.M., Zamolodchikov, A.B.: Infinite conformal symmetry in two-dimensional quantum field theory. Nucl. Phys. B 241, 333-380 (1984)

4. van de Bult, F.J.: Ruijsenaars' hypergeometric function and the modular double of $U_{q}(\operatorname{sl}(2, C))$. Adv. Math. 204, 53971 (2006)

5. van de Bult, F.J., Rains, E.M., Stokman, J.V.: Properties of generalized univariate hypergeometric functions. Commun. Math. Phys. 275, 3795 (2007)

6. Collier, S., Gobeil, Y., Maxfiel, H., Perlmutter, E.: Quantum Regge trajectories and the Virasoro analytic bootstrap. J. High Energy Phys. 2019, 212 (2019)

7. Chang, C.M., Lin, Y.H.: Bootstrap, universality and horizons. J. High Energy Phys. 2016, 68 (2016)

8. Drukker, N., Gomis, J., Okuda, T., Teschner, J.: Gauge theory loop operators and liouville theory. J. High Energy Phys. 2010, 57 (2010)

\footnotetext{
4 A similar identity using a degeneration limit of the Spiridonov's $V$-function was obtained in [38].
} 
9. Faddeev, L., Kashaev, R.: Quantum dilogarithm. Mod. Phys. Lett. 9, 265-282 (1994)

10. Hadasz, L., Jaskolski, Z., Piatek, M.: Analytic continuation formulae for the BPZ conformal blocks. Acta Phys. Polon. B 36, 845-864 (2005)

11. Hadasz, L., Jaskólski, Z., Suchanek, P.: Modular bootstrap in Liouville field theory. Phys. Lett. B 685 , 79 (2010)

12. Kusuki, Y.: Light cone bootstrap in general 2D CFTs and entanglement from light cone singularity. J. High Energy Phys. 2019, 25 (2019)

13. Koornwinder, T.H.: Askey-Wilson polynomials for root systems of type $B C$, in: Hypergeometric functions on domains of positivity, Jack polynomials, and applications (Tampa, FL, 1991), 189204 , Contemp. Math., 138, Am. Math. Soc., Providence, RI (1992)

14. Koornwinder, T.H., Mazzocco, M.: Dualities in the q-Askey scheme and degenerate DAHA. Stud. Appl. Math. 141, 424473 (2018)

15. Lenells, J., Roussillon, J.: Confluent conformal blocks of the second kind. J. High Energy Phys. 2020, 133 (2020)

16. Moore, G., Seiberg, N.: Classical and quantum conformal field theory. Commun. Math. Phys. 123, 177-254 (1989)

17. Nemkov, N.A.: Fusion transformations in Liouville theory. Theo. Math. Phys. 189, 1574-1591 (2016)

18. Nemkov, N.A.: On modular transformations of toric conformal blocks. J. High Energy Phys. 2015, 39 (2015)

19. Noumi, M., Stokman, J.V.: Askey-Wilson polynomials: an affine Hecke algebra approach, Laredo Lectures on Orthogonal Polynomials and Special Functions, Adv. Theory Spec. Funct. Orthogonal Polynomials, Nova Science Publication, Hauppauge, NY, 2004, pp. 111144. MR 2085854 (2005h:42057)

20. Nidaiev, I., Teschner, J.: On the relation between the modular double of $U_{q}(\operatorname{sl}(2, R))$ and the quantum Teichmüller theory. arXiv:1302.3454 [math-ph]

21. Ponsot, B., Teschner, J.: Liouville bootstrap via harmonic analysis on a noncompact quantum group. arXiv:hep-th/9911110

22. Ponsot, B., Teschner, J.: Clebsch-Gordan and Racah-Wigner coefficients for a continuous series of representations of $\mathcal{U}_{q}(\mathfrak{s l}(2, \mathbb{R}))$. Commun. Math. Phys. 224, 613-655 (2001)

23. Ribault, S.: Conformal field theory on the plane. arXiv:1406.4290v5 [hep-th]

24. Ruijsenaars, S.: Systems of Calogero-Moser type, Particles and fields, 251-352

25. Ruijsenaars, S.: First order analytic difference equations and integrable quantum systems. J. Math. Phys. 38, 1069 (1997)

26. Ruijsenaars, S.: A generalized hypergeometric function satisfying four analytic difference equations of Askey-Wilson type. Commun. Math. Phys. 206, 639-690 (1999)

27. Ruijsenaars, S.: A generalized hypergeometric function II. Asymptotics and D4 Symmetry, Commun. Math. Phys. 243, 389412 (2003)

28. Ruijsenaars, S.: A generalized hypergeometric function III. Associated Hilbert Space Transform, Commun. Math. Phys. 243, 413448 (2003)

29. Ruijsenaars, S.: A relativistic conical function and its Whittaker Limits, SIGMA 7 (2011), 101, 54 pages

30. Ruijsenaars, S.N.M.: Complete integrability of relativistic Calogero-Moser systems and elliptic function identities. Commun. Math. Phys. 110, 191213 (1987)

31. Ruijsenaars, S.N.M., Schneider, H.: A new class of integrable systems and its relation to solitons. Ann. Phys. (NY) 170, 370405 (1986)

32. Spiridonov, V.P.: Theta hypergeometric integrals. Algebra i Analiz 15(6), 161-215 (2003) (St. Petersburg Math. J. 15(6), 929-967 (2004))

33. Schrader, G., Shapiro, A.: Continuous tensor categories from quantum groups I: algebraic aspects. arXiv:1708.08107 [math.RT]

34. Schrader, G., Shapiro, A.: On b-Whittaker functions. arXiv:1806.00747 [math-ph]

35. Teschner, J.: Liouville theory revisited, Class. Quant. Grav. 18, R153R222 (2001)

36. Teschner, J.: A lecture on Liouville vertex operators, Int. J. Mod. Phys. A 19S2, 436458 (2004)

37. Teschner, J., Vartanov, G.: Supersymmetric gauge theories, Quantization of $\mathcal{M}_{\text {flat }}$, and conformal field theory. arXiv:1302.3778 [hep-th]

38. Teschner, J., Vartanov, G.: $6 \mathrm{j}$ symbols for the modular double, quantum hyperbolic geometry, and supersymmetric gauge theories. Lett. Math. Phys. 104, 527551 (2014) 
39. Terashima, Y., Yamazaki, M.: SL( $2, \mathbb{R})$ Chern-Simons, Liouville, and gauge theory on duality walls. J. High Energy Phys. 2011, 135 (2011)

40. van Diejen, J.F.: Integrability of difference Calogero-Moser systems. J. Math. Phys. 35, 2983 (1994)

41. Woronowicz, S.L.: Quantum exponential function. Rev. Math. Phys. 12, 873-920 (2000)

42. Al, B. Zamolodchikov.: Conformal symmetry in two-dimensional space: Recursion representation of conformal block, TMF 73, : 103-110. Theor. Math. Phys. 73(1987), 1088-1093 (1987)

Publisher's Note Springer Nature remains neutral with regard to jurisdictional claims in published maps and institutional affiliations. 